\title{
THE MASS DISTRIBUTION AND LIFETIME OF PRESTELLAR CORES IN PERSEUS, SERPENS, AND OPHIUCHUS
}

\author{
Melissa L. Enoch, ${ }^{1,2}$ Neal J. Evans II, ${ }^{3}$ Anneila I. Sargent, ${ }^{2}$ Jason Glenn, $^{4}$ \\ ERIK Rosolowsky, ${ }^{5,6}$ AND PhILIP Myers ${ }^{6}$ \\ Received 2008 February 1; accepted 2008 May 7
}

\begin{abstract}
We present an unbiased census of starless cores in Perseus, Serpens, and Ophiuchus, assembled by comparing largescale Bolocam $1.1 \mathrm{~mm}$ continuum emission maps with Spitzer c2d surveys. We use the c2d catalogs to separate 108 starless from 92 protostellar cores in the $1.1 \mathrm{~mm}$ core samples from Enoch and Young and their coworkers. A comparison of these populations reveals the initial conditions of the starless cores. Starless cores in Perseus have similar masses but larger sizes and lower densities on average than protostellar cores, with sizes that suggest density profiles substantially flatter than $\rho \propto r^{-2}$. By contrast, starless cores in Serpens are compact and have lower masses than protostellar cores; future star formation will likely result in lower mass objects than the currently forming protostars. Comparison to dynamical masses estimated from the $\mathrm{NH}_{3}$ survey of Perseus cores by Rosolowsky and coworkers suggests that most of the starless cores are likely to be gravitationally bound, and thus prestellar. The combined prestellar core mass distribution includes 108 cores and has a slope of $\alpha=-2.3 \pm 0.4$ for $M>0.8 M_{\odot}$. This slope is consistent with recent measurements of the stellar initial mass function, providing further evidence that stellar masses are directly linked to the core formation process. We place a lower limit on the core-to-star efficiency of $25 \%$. There are approximately equal numbers of prestellar and protostellar cores in each cloud; thus the dense prestellar core lifetime must be similar to the lifetime of embedded protostars, or $4.5 \times 10^{5} \mathrm{yr}$, with a total uncertainty of a factor of 2. Such a short lifetime suggests a dynamic, rather than quasi-static, core evolution scenario, at least at the relatively high mean densities $\left(n>2 \times 10^{4} \mathrm{~cm}^{-3}\right)$ to which we are sensitive.
\end{abstract}

Subject headings: infrared: ISM - ISM: clouds — ISM: individual (Ophiuchus, Perseus, Serpens) stars: formation - submillimeter

Online material: color figures, machine-readable tables

\section{INTRODUCTION}

Dense prestellar cores, from which a new generation of stars will form, represent a very early stage of the low-mass star formation process, before collapse results in the formation of a central protostar. The mass and spatial distributions of these prestellar cores retain imprints of their formation process, and their lifetime is extremely sensitive to the dominant physics controlling their formation. It is well established that most of the star formation in our Galaxy occurs in clusters and groups within large molecular clouds (e.g., Lada \& Lada 2003 and references therein). Molecular clouds are known to be turbulent, with supersonic line widths (e.g., McKee \& Zweibel 1992), and to have complex magnetic fields that are likely important to the cloud physics (e.g., Crutcher 1999). Understanding the properties of prestellar cores on molecular cloud scales, and how they vary with environment, provides insight into the global physical processes controlling star formation in molecular clouds.

For example, in the classic paradigm of magnetically dominated star formation (Shu et al. 1987), the collapse of cores occurs

\footnotetext{
${ }^{1}$ Department of Astronomy, University of California, Berkeley, CA 94720; menoch@astro.berkeley.edu.

${ }^{2}$ Division of Physics, Mathematics, and Astronomy, California Institute of Technology, Pasadena, CA 91125.

${ }^{3}$ University of Texas, Astronomy Department, 1 University Station C1400, Austin, TX 78712-0259.

${ }^{4}$ Center for Astrophysics and Space Astronomy, 389-UCB, University of Colorado, Boulder, CO 80309.

5 University of British Columbia, Okanagan, 3333 University Way, Kelowna BC V1V 1V7, Canada.

${ }^{6}$ Harvard-Smithsonian Center for Astrophysics, 60 Garden Street, Cambridge, MA 02138
}

very slowly via ambipolar diffusion and starless cores should be long lived, with lifetimes of order $t_{\mathrm{AD}} \sim 10 t_{\mathrm{ff}}$ (Nakano 1998), where $t_{\mathrm{ff}}$ is the free-fall timescale $\left(t_{\mathrm{ff}} \sim 10^{5}\right.$ yr for $n \sim 10^{5} \mathrm{~cm}^{-3}$, where $t_{\mathrm{ff}}$ depends on the mean core density $n: t_{\mathrm{ff}} \propto n^{-0.5}$ ). Alternatively, if molecular cloud evolution is driven primarily by turbulence, overdense cores should collapse quickly, on approximately a dynamical timescale, $(1-2) t_{\mathrm{ff}}$ (Ballesteros-Paredes et al. 2003; Mac Low \& Klessen 2004). Thus, the lifetime of prestellar cores should be a strong discriminator of core formation mechanisms. Published measurements of the prestellar core lifetime vary by 2 orders of magnitude, however, from a few times $10^{5}$ to $10^{7} \mathrm{yr}$ (see Ward-Thompson et al. 2007 and references therein).

While the properties of star-forming cores depend strongly on the physical processes leading to their formation, core initial conditions in turn help to determine the evolution of newly formed protostars. One of the most important diagnostics of initial conditions is the mass distribution of prestellar cores. In addition to being a testable prediction of core formation models, a comparison of the core mass distribution (CMD) to the stellar initial mass function (IMF) may reveal what process is responsible for determining stellar masses (e.g., Meyer et al. 2000).

A number of recent studies have found observational evidence that the shape of the IMF is directly tied to the core fragmentation process (Testi \& Sargent 1998; Motte et al. 1998; Onishi et al. 2002). Alves et al. (2007) find a turnover in the CMD of the Pipe Nebula at $M \sim 3$ times the turnover in the Trapezium IMF (Muench et al. 2002), and suggest that the stellar IMF is a direct product of the CMD, with a uniform core-to-star efficiency of $30 \% \pm 10 \%$. The mean particle densities of the extinctionidentified cores in the Pipe Nebula study $\left(5 \times 10^{3}-2 \times 10^{4} \mathrm{~cm}^{-3}\right)$ are lower than those of typical cores traced by dust emission 
$\left(2 \times 10^{4}-10^{6} \mathrm{~cm}^{-3} ;^{7}\right.$ Enoch et al. 2007), however, and the cores may not be truly prestellar. In Orion, Nutter \& Ward-Thompson (2007) find a turnover in the CMD of starless SCUBA $850 \mu \mathrm{m}$ cores at $\sim 1.3 M_{\odot}$. Those authors relate this turnover to a downturn in the Kroupa (2002) IMF at $\sim 0.1 M_{\odot}$, and infer a much lower core-to-star efficiency of $6 \%$.

Large samples of prestellar cores are important for further addressing these problems, as is a more reliable separation of prestellar, protostellar, and unbound starless cores. We follow Di Francesco et al. (2007) in defining starless cores as low-mass dense cores without a compact internal luminosity source, and prestellar cores, at least conceptually, as starless cores that are gravitationally bound and will form stars in the future. For millimeter cores containing a compact luminous internal source (i.e., an embedded protostar) we follow Di Francesco et al. (2007) in terming these protostellar cores, regardless of whether the final object will be stellar or substellar in nature. Unlike protostellar cores, which are internally heated by the embedded source as well as externally by the interstellar radiation field (ISRF), starless cores are heated only externally by the ISRF, with decreasing temperatures toward the core center (e.g., Evans et al. 2001).

Molecular line or extinction surveys often trace relatively low density material $\left(10^{3}-10^{4} \mathrm{~cm}^{-3}\right)$, leaving the possibility that such cores may never collapse to form stars. In addition, most previous studies base the identification of protostellar versus starless cores on near-infrared data, which are not sensitive to the most embedded protostars, or on low-resolution and poor-sensitivity IRAS maps. The first issue can be remedied by using millimeter or submillimeter surveys; (sub)millimeter emission traces dense $(n \gtrsim$ $2 \times 10^{4} \mathrm{~cm}^{-3}$; Ward-Thompson et al. 1994; Enoch et al. 2007) material, and detection at (sub)millimeter wavelengths tends to correlate with other indications of a prestellar nature, such as inward motions (Gregersen \& Evans 2000). Spitzer provides significant progress on the second issue, with substantially superior resolution and sensitivity $\left(0.01 L_{\odot}\right.$ at $260 \mathrm{pc}$; Harvey et al. $2007 \mathrm{~b}$ ) compared to $I R A S$, making the identification of prestellar cores much more secure. Recent examples of identifying starless cores include the studies of Jørgensen et al. (2007) and Hatchell et al. (2007) which utilize SCUBA $850 \mu \mathrm{m}$ surveys and Spitzer data to distinguish starless from protostellar cores in the Perseus molecular cloud.

The combination of(sub)millimeter studies with molecular line observations of dense gas tracers, which yield a gas temperature and line width, is a powerful method for determining the mechanical balance of starless cores. While our preliminary operational definition of a prestellar core will be a starless core that is detected at submillimeter or millimeter wavelengths, we will examine this issue more closely in $\S 4$. Comparison of our data to molecular line observations, such as the recent $\operatorname{GBT~NH}_{3}(2,2)$ and $(1,1)$ survey of Bolocam cores in Perseus by Rosolowsky et al. (2008), provides a more robust method of estimating whether cores are gravitationally bound.

We have recently completed large continuum surveys at $\lambda=$ $1.1 \mathrm{~mm}$ of the Perseus, Ophiuchus, and Serpens molecular clouds using Bolocam at the Caltech Submillimeter Observatory (CSO). We mapped $7.5 \mathrm{deg}^{2}\left(140 \mathrm{pc}^{2}\right.$ at our adopted cloud distance of $d=250 \mathrm{pc})$ in Perseus, $10.8 \operatorname{deg}^{2}\left(50 \mathrm{pc}^{2}\right.$ at $\left.d=125 \mathrm{pc}\right)$ in Ophiuchus, and $1.5 \mathrm{deg}^{2}\left(30 \mathrm{pc}^{2}\right.$ at $\left.d=260 \mathrm{pc}\right)$ in Serpens with a resolution of 31" (Enoch et al. 2006; Young et al. 2006; Enoch et al. 2007; hereafter Papers I, II, and III, respectively). Millimeter

\footnotetext{
7 The mean particle density of Bolocam cores is given by $n=3 M /\left(4 \pi R \mu m_{\mathrm{H}}\right)$, where $M$ is the core mass, $R$ the radius, $\mu=2.33$ the mean molecular weight per particle, and $m_{\mathrm{H}}$ the mass of hydrogen.
}

emission traces the properties of starless cores and protostellar envelopes, including core sizes, shapes, masses, densities, and spatial distribution. The $1.1 \mathrm{~mm}$ Bolocam surveys are complemented by large Spitzer Space Telescope IRAC and MIPS maps of the same clouds from the "From Molecular Cores to Planet-forming Disks" Spitzer Legacy program ("Cores to Disks" or c2d; Evans et al. 2003). Combining these data sets with the 2MASS survey provides wavelength coverage from 1.25 to $160 \mu \mathrm{m}$, and enables us to reliably differentiate starless cores from those that have already formed embedded protostars. The Spitzer c2d IRAC and MIPS surveys of each cloud are described in detail in Jørgensen et al. (2006), Harvey et al. (2006, 2007b), Rebull et al. (2007), and Padgett et al. (2008).

In Paper III, we looked at how the global molecular cloud environment influences the properties of star-forming cores, by comparing the $1.1 \mathrm{~mm}$ core populations in Perseus, Serpens, and Ophiuchus. In a companion paper to this work (M. L. Enoch et al. 2008, in preparation), we use the comparison of $1.1 \mathrm{~mm}$ and Spitzer data to study the properties of embedded protostars. In particular, we examine the bolometric temperatures and luminosities of Class 0 and Class I protostars in Perseus, Serpens, and Ophiuchus, the lifetime of the Class 0 phase, and accretion rates and history for the early protostellar phases. Here we use the combination of Bolocam $1.1 \mathrm{~mm}$ and Spitzer $\mathrm{c} 2 \mathrm{~d}$ surveys to probe the initial conditions of star formation on molecular cloud scales, and how the properties of starless cores differ from cores that have already formed protostars.

In $\S 2$ we describe the identification of protostellar cores, including the combination of $1.1 \mathrm{~mm}$ and Spitzer infrared (IR) data ( $\$ 2.1$ ), identification of candidate protostars based on their midand far-infrared properties ( $\S 2.2$ ), and the basis on which we determine association between $1.1 \mathrm{~mm}$ cores and candidate protostars $(\S 2.3)$. The resulting starless and protostellar $1.1 \mathrm{~mm}$ core populations for each cloud are compared in $\S 3$, including core sizes and shapes $(\S 3.1)$, masses and densities $(\S 3.2)$, distribution in mass-versus-size $(\S 3.3)$, relationship to cloud column density ( $\S 3.4$ ), and spatial clustering ( $\S 3.5)$. We calculate the dynamical mass of starless cores in Perseus using $\mathrm{NH}_{3}$ observations to determine whether the cores are truly prestellar $(\S 4)$. We combine the three clouds to produce the prestellar CMD, which is discussed in relation to the stellar IMF in $\S 5$. Finally, in $\S 6$ we estimate the lifetime of the dense prestellar core phase and discuss implications for star formation theory.

\section{SEPARATING STARLESS AND PROTOSTELLAR CORES}

To study the initial conditions of star formation as traced by prestellar cores, we first must differentiate cores without an internal source of luminosity (starless cores) from those with an embedded self-luminous source (protostellar cores). Protostellar cores will have lost some mass due to accretion onto the embedded protostar, and may be otherwise altered, so that they are no longer representative of core initial conditions. Starless and protostellar cores can be differentiated using the Spitzer c2d surveys, by identifying infrared sources that may be associated with a given core. Such candidate protostars are typically visible as pointlike objects in the near- to mid-infrared data. In the following sections we describe the merging of the millimeter and infrared data and the criteria used to determine which cores are protostellar.

\subsection{Combining Bolocam and Spitzer c2d Data}

Spitzer IRAC and MIPS maps from the c2d Legacy program cover nearly the same area as our Bolocam $1.1 \mathrm{~mm}$ maps of Perseus, Serpens, and Ophiuchus. Both Bolocam and Spitzer 
maps were designed to cover down to a visual extinction of $A_{V} \gtrsim$ 2 mag in Perseus, $A_{V} \gtrsim 3$ mag in Ophiuchus, and $A_{V} \gtrsim 6$ mag in Serpens (Evans et al. 2003). The actual overlap in area between Bolocam and IRAC maps is shown in Figure 1 of Papers I, II, and III for Perseus, Ophiuchus, and Serpens, respectively. Catalogs listing c2d Spitzer fluxes of all detected sources in each of the three clouds, as well as near-infrared fluxes for sources that also appear in the 2MASS catalogs, are available through the Spitzer database (Evans et al. 2007). Thus, we have wavelength coverage from $\lambda=1.25$ to $1100 \mu \mathrm{m}$, utilizing $2 \mathrm{MASS}(\lambda=1.25,1.65$, $2.17 \mu \mathrm{m}), \operatorname{IRAC}(\lambda=3.6,4.5,5.8,8.0 \mu \mathrm{m}), \operatorname{MIPS}(\lambda=24,70$, $160 \mu \mathrm{m})$, and Bolocam $(\lambda=1.1 \mathrm{~mm})$ data. Note that $160 \mu \mathrm{m}$ flux measurements are not included in the $\mathrm{c} 2 \mathrm{~d}$ delivery catalogs due to substantial uncertainties and incompleteness, but are utilized here and in M. L. Enoch et al. (2008, in preparation) when possible.

Basic data papers describe the processing and analysis of the Spitzer IRAC and MIPS maps of Perseus, Serpens, and Ophiuchus, and present general properties of the sources in each cloud such as color-color and color-magnitude diagrams (Jørgensen et al. 2006; Harvey et al. 2006, 2007b; Rebull et al. 2007). In addition, the young stellar object (YSO) population in Serpens is discussed in detail by Harvey et al. (2007a). Here we are most interested in very red sources that are likely to be embedded in the millimeter cores detected with Bolocam. For the following we will use the term "candidate protostar" in general to encompass candidate Class 0 and Class I objects (André et al. 1993; Lada \& Wilking 1984), although more evolved sources may be included in this sample as well. A more detailed study of the properties of the candidate protostars themselves is carried out in a companion paper (M. L. Enoch et al. 2008, in preparation).

In Figure 1 we show the result of combining Spitzer and Bolocam data for a few cores in each cloud. Three-color $(8,24$, $160 \mu \mathrm{m})$ Spitzer images are overlaid with $1.1 \mathrm{~mm}$ Bolocam contours, and symbols mark the positions of all identified $1.1 \mathrm{~mm}$ starless cores (crosses) and protostellar cores (squares). Bolocam IDs of the central sources, from Table 1 of Papers I-III, are given at the top of each image. Red $160 \mu \mathrm{m}$ images are often affected by saturation, pixel artifacts (bright pixels), and incomplete coverage. Saturation by bright sources affects many pixels in a given scan; this and incomplete sampling accounts for the striped appearance of the red images, particularly in Ophiuchus.

Note the lack of infrared point sources near the center of the starless $1.1 \mathrm{~mm}$ cores, whereas protostellar $1.1 \mathrm{~mm}$ cores are clearly associated with one or more Spitzer sources. Apart from these common traits, both starless and protostellar cores display a wide range of properties. They may be isolated single sources (e.g., Per-Bolo 62, Oph-Bolo 42), associated with filaments or groups (Per-Bolo 74, Ser-Bolo 20), or found near very bright protostars (Per-Bolo 57). Although most of the starless cores are extended, some are quite compact (e.g., Per-Bolo 57), barely resolved by the $31^{\prime \prime}$ Bolocam beam. Some starless cores are distinguished in the Spitzer bands by their bright $160 \mu \mathrm{m}$ emission. Per-Bolo 62 is not detectable at 24 or $70 \mu \mathrm{m}$, but emerges as a diffuse source at $160 \mu \mathrm{m}$, indicating a cold, extended core that closely mirrors $1.1 \mathrm{~mm}$ contours. Other starless cores stand out as dark "shadows" in the shorter wavelength bands. Ser-Bolo 18 and Oph-Bolo 26 are two examples of such cores; the dark shadows against bright 8 and $24 \mu \mathrm{m}$ emission are suggestive of dense cores obscuring background nebular emission. Again, contours at $1.1 \mathrm{~mm}$ closely trace the Spitzer short-wavelength shadows.

Although the sky coverage of the IRAC, MIPS, and Bolocam maps overlaps nearly perfectly for our purposes, there is a small portion of the Serpens $1.1 \mathrm{~mm}$ map that is not covered by the $70 \mu \mathrm{m}$ map. In addition, the $160 \mu \mathrm{m}$ maps are often saturated near bright sources and in regions of bright extended emission, such as near bright clusters of sources.

\subsection{Identifying Candidate Protostars}

The identification of candidate protostars is discussed in more detail in M. L. Enoch et al. (2008, in preparation); here we briefly describe the sample used to identify protostellar cores. The first criteria used to select candidate protostars from the $\mathrm{c} 2 \mathrm{~d}$ source catalogs is based on the source "class." All sources in the c2d database are assigned a class parameter based on colors, magnitudes, and stellar SED fits, as discussed in the c2d Delivery Document (Evans et al. 2007) and in Harvey et al. (2007b). Class parameters include "star," "star+disk," "YSOc" (young stellar object candidate), "red," "rising," "Galc" (galaxy candidate), etc. Protostar candidates will generally be a subset of YSOc sources, but some of the most embedded may also be assigned to the "red" class if they are not detected in all four IRAC bands.

A preliminary sample is formed from sources classified as "YSOc" or "red." We impose a flux limit at $\lambda=24 \mu \mathrm{m}$ of $S_{24 \mu \mathrm{m}} \geq 3 \mathrm{mJy}^{8}$, high enough to eliminate most extragalactic interlopers and sources with SEDs that are clearly inconsistent with an embedded nature (e.g., sources with $S_{24 \mu \mathrm{m}}<S_{8 \mu \mathrm{m}}$ ), but low enough to include most deeply embedded known protostars. In addition, we include any $70 \mu \mathrm{m}$ point sources that are not classified as galaxy candidates ("Galc"). In each cloud, a few known deeply embedded sources that have strong $70 \mu \mathrm{m}$ emission but very weak $24 \mu \mathrm{m}$ emission (e.g., HH 211 in Perseus) are recovered by this last criteria, as are a few very bright sources that are saturated at $24 \mu \mathrm{m}$ (these are often classified as "rising"). The Spitzer c2d surveys are complete to young objects with luminosities as low as $0.05 L_{\odot}$ (Dunham et al. 2008; Harvey et al. $2007 \mathrm{~b}$ ), and we are unlikely to be missing any protostellar sources down to this level (M. L. Enoch et al. 2008, in preparation; Dunham et al. 2008).

\subsection{Determining Association between Cores and Candidate Protostars}

We next determine which of the $1.1 \mathrm{~mm}$ cores are associated with a candidate protostar. The positional offset from each candidate protostar to the nearest $1.1 \mathrm{~mm}$ core centroid position, in units of the core full width at half-maximum (FWHM) size, is plotted in Figure 2 (left). Note that this analysis is similar to Figure 2 of Jørgensen et al. (2007). Large circles enclose candidate protostars that are located within $0.5 \times \theta_{1 \mathrm{~mm}}$ and $1.0 \times$ $\theta_{1 \mathrm{~mm}}$ of a $1.1 \mathrm{~mm}$ core position, where $\theta_{1 \mathrm{~mm}}$ is the angular FWHM size of a given core (the measurement of core sizes is described in $\S 3.1$ ). Protostellar sources with bolometric temperature $T_{\text {bol }}<300 \mathrm{~K}$ (M. L. Enoch et al. 2008, in preparation) are indicated by bold symbols. In general, the coldest objects, those expected to be embedded within millimeter cores, are located within $1.0 \times \theta_{1 \mathrm{~mm}}$ of a $1.1 \mathrm{~mm}$ core position. Thus we define protostellar cores to be those cores which have a candidate protostar located within $1.0 \times \theta_{1 \mathrm{~mm}}$ of the core center. Note that protostellar cores are defined by any candidate protostar within $1.0 \times \theta_{1 \mathrm{~mm}}$, not only those with $T_{\text {bol }}<300 \mathrm{~K}$.

The right panel of Figure 2 demonstrates what we would expect if the same sample of Spitzer sources was distributed randomly

\footnotetext{
${ }^{8}$ Note that this limit is considerably higher than the $S_{24 \mu \mathrm{m}} \gtrsim 0.7 \mathrm{mJy}$ limit imposed by Harvey et al. (2007a) together with several other criteria, to help eliminate galaxies.
} 

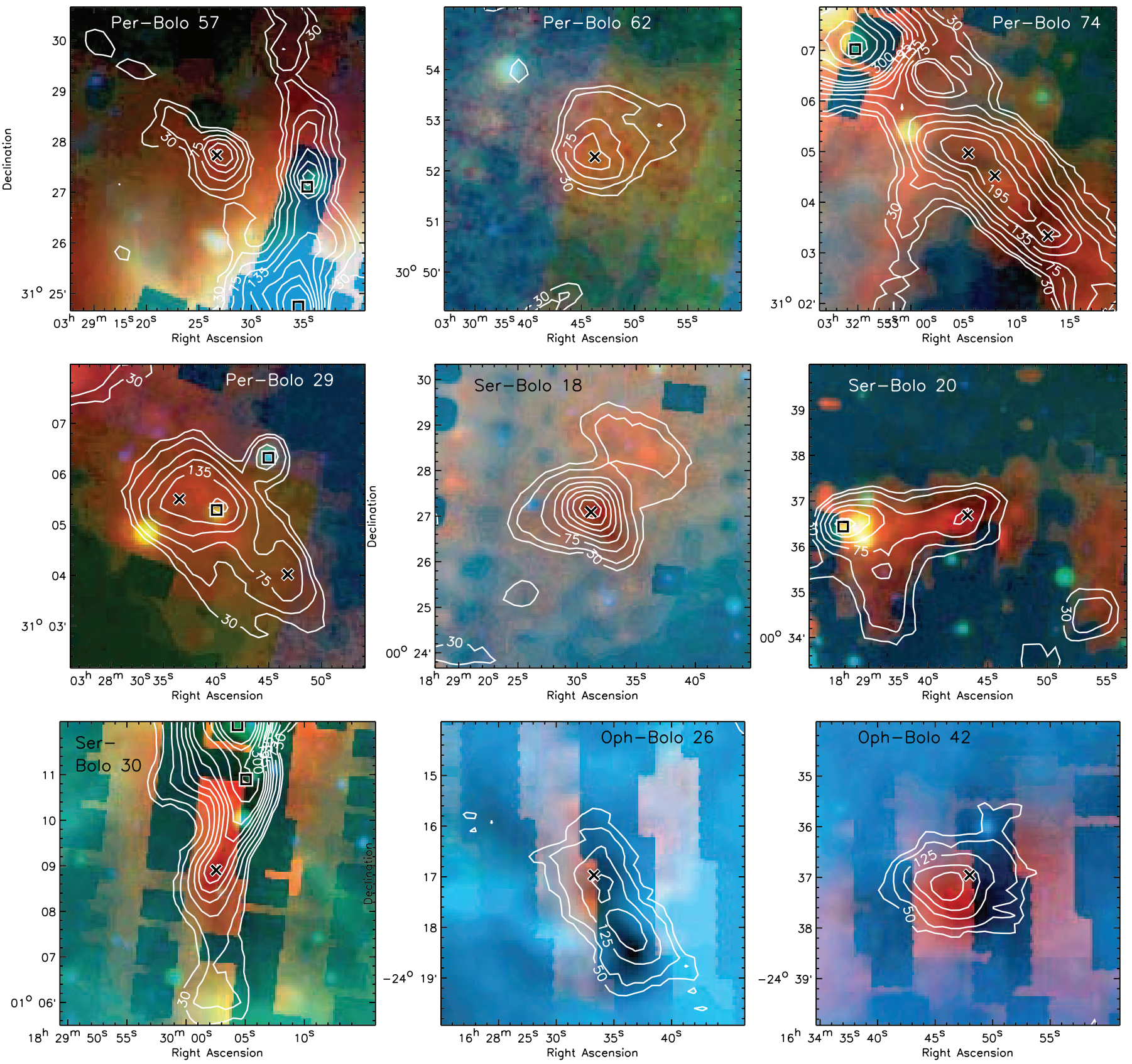

FIG. 1.- Three-color Spitzer images $(8.0,24,160 \mu \mathrm{m})$ of selected starless and protostellar cores in Perseus, Serpens, and Ophiuchus. Bolocam $1.1 \mathrm{~mm}$ contours are overlaid at intervals of $2,3,5, \ldots, 15,20, \ldots, 35 \sigma$, where $1 \sigma$ is the mean rms noise in each $1.1 \mathrm{~mm}$ Bolocam map ( $15 \mathrm{mJy}$ in Perseus, $10 \mathrm{mJy}$ in Serpens, and $25 \mathrm{mJy}$ in Ophiuchus).

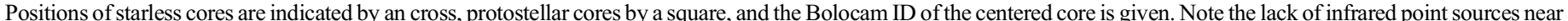

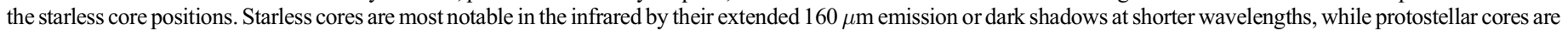
clearly associated with Spitzer point sources. Saturation, image artifacts, and incomplete sampling causes the striped appearance of some of the red $160 \mu \mathrm{m}$ images.

over the Bolocam map area. Although there are approximately the same number of sources in the random sample as in the candidate protostar sample, most do not appear on the plot because they are located much farther than $2 \times \theta_{1 \mathrm{~mm}}$ from the nearest core. There are five sources from the random sample located within $1.0 \times$ $\theta_{1 \mathrm{~mm}}$ of a core position; thus we can expect a few false associations between $1.1 \mathrm{~mm}$ cores and candidate protostars based on this criteria. Indeed, some associations between cores and Spitzer sources are probably just projections on the sky. We are especially skeptical of associations where the $1.1 \mathrm{~mm}$ core flux density is much higher than the $70 \mu \mathrm{m}$ flux. Using $0.5 \times \theta_{1 \mathrm{~mm}}$ would be a more restrictive choice, but might misidentify some protostellar cores as starless. As we do not want to contaminate our starless core sample with more evolved sources, we adopt the more conservative criteria. Thus the number of starless cores in each cloud is likely a lower limit to the true value; using $0.5 \times \theta_{1 \mathrm{~mm}}$ would result in three more starless cores in Perseus, two in Serpens, and four in Ophiuchus.

\section{COMPARING THE STARLESS AND PROTOSTELLAR $1.1 \mathrm{~mm}$ CORE POPULATIONS}

Tables 1 and 2 list the Bolocam identifications, positions, and peak flux densities (from Papers I-III) of starless and protostellar cores in each cloud. We find a total of 108 starless and 92 protostellar cores in the three cloud sample. Cores are identified based on a peak flux density at least 5 times the local rms noise 

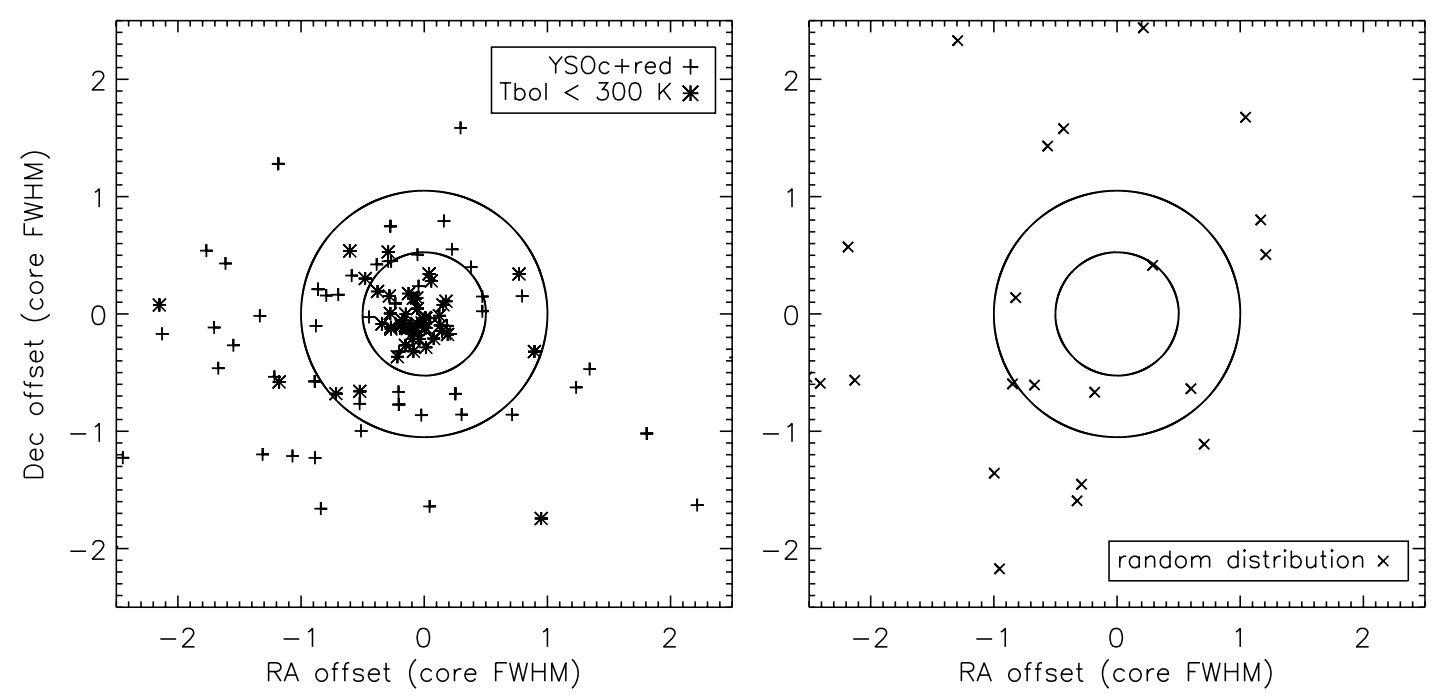

FIG. 2.-Left: Distribution of the positional offset from each protostar candidate in Perseus to the nearest $1.1 \mathrm{~mm}$ core centroid. Distances are in units of the core FWHM size $\left(\theta_{1 \mathrm{~mm}}\right)$, and circles enclose sources within 0.5 and $1.0 \times \theta_{1 \mathrm{~mm}}$ of a core position. Pointing errors in the Bolocam maps are typically $10^{\prime \prime}$ or less. Protostar candidates include all "YSOc," "red," and $70 \mu \mathrm{m}$ sources, while candidates with bolometric temperatures $T_{\text {bol }}<300 \mathrm{~K}$ (M. L. Enoch et al. 2008, in preparation) are shown in bold. These cold objects, which are most likely to be most embedded, are primarily located within $1.0 \times \theta_{1 \mathrm{~mm}}$ of a core centroid. Right: Expected distribution if the IR sources were distributed randomly over the Bolocam map area (i.e., if they were not spatially correlated with the cores). Our definition that a given $1.1 \mathrm{~mm}$ core is protostellar if there is a candidate protostar within $1.0 \times \theta_{1 \mathrm{~mm}}$ of the core position will result in a few false associations in each cloud.

TABLE 1

Starless Cores in Perseus, Serpens, and Ophiuchus

\begin{tabular}{|c|c|c|c|c|c|c|c|}
\hline Bolocam ID & $\begin{array}{c}\text { R.A. } \\
(\mathrm{J} 2000.0)\end{array}$ & $\begin{array}{c}\text { Decl. } \\
(\mathrm{J} 2000.0)\end{array}$ & $\begin{array}{c}\text { Peak Flux } \\
\left(\mathrm{mJy} \text { beam }^{-1}\right)\end{array}$ & $\begin{array}{l}\text { Total Mass } \\
\qquad\left(M_{\odot}\right)\end{array}$ & $\begin{array}{c}\theta_{\mathrm{dec}} \\
(\operatorname{arcsec})\end{array}$ & Axis Ratio & $\begin{array}{c}n_{1 e 4} \\
\left(\mathrm{~cm}^{-3}\right)\end{array}$ \\
\hline \multicolumn{8}{|c|}{ Perseus } \\
\hline Per-Bolo 1 ..................... & 032507.8 & +302422 & 109 & 0.28 & 30.4 & 1.5 & $0.9 \times 10^{5}$ \\
\hline 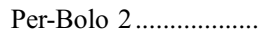 & 032509.5 & +302351 & 121 & 0.36 & 15.7 & 1.6 & $1.1 \times 10^{5}$ \\
\hline 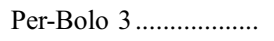 & 032510.2 & +304443 & 125 & 0.92 & 85.5 & 2.1 & $1.0 \times 10^{5}$ \\
\hline Per-Bolo 4 .................... & 032517.0 & +301853 & 149 & 2.99 & 104.9 & 1.2 & $1.5 \times 10^{5}$ \\
\hline Per-Bolo $6 \ldots \ldots \ldots \ldots . . . .$. & 032526.5 & +302150 & 143 & 2.85 & 105.6 & 1.4 & $1.3 \times 10^{5}$ \\
\hline Per-Bolo 7 ..................... & 032535.4 & +301306 & 126 & 2.32 & 98.1 & 1.4 & $1.1 \times 10^{5}$ \\
\hline Per-Bolo 9 ..................... & 032537.2 & +300955 & 113 & 1.70 & 98.2 & 1.9 & $1.0 \times 10^{5}$ \\
\hline Per-Bolo 11 ..................... & 032546.0 & +304410 & 241 & 2.18 & 72.5 & 1.0 & $2.2 \times 10^{5}$ \\
\hline Per-Bolo $12 \ldots \ldots \ldots \ldots \ldots . . . . . . .$. & 032547.5 & +301226 & 118 & 2.34 & 95.6 & 1.8 & $1.1 \times 10^{5}$ \\
\hline
\end{tabular}

Notes.-Units of right ascension are hours, minutes, and seconds, and units of declination are degrees, arcminutes, and arcseconds. Bolocam identification is from Papers I-III. The total mass is calculated from the total flux at $1.1 \mathrm{~mm}$, assuming $T_{D}=10 \mathrm{~K}$. Deconvolved angular FWHM sizes $\left(\theta_{\mathrm{dec}}\right)$ and axis ratios are derived from an elliptical Gaussian fit, deconvolved by the $31^{\prime \prime}$ beam. Mean densities are calculated in a fixed linear aperture of diameter $10^{4} \mathrm{AU}$ for each source. Note that the Ophiuchus identifications are different than in Tables 1 and 2 of Paper II for Oph-Bolo 35 and above due to an adjustment of the source list, resulting in 43 rather than 44 sources (IDs are shifted by -1 for Oph-Bolo 35 and up). Table 1 is published in its entirety in the electronic edition of the Astrophysical Journal. A portion is shown here for guidance regarding its form and content.

TABLE 2

Protostellar Cores in Perseus, Serpens, and Ophiuchus

\begin{tabular}{|c|c|c|c|c|c|c|c|}
\hline Bolocam ID & $\begin{array}{c}\text { R.A. } \\
\text { (J2000.0) }\end{array}$ & $\begin{array}{c}\text { Decl. } \\
(\mathrm{J} 2000.0)\end{array}$ & $\begin{array}{c}\text { Peak Flux } \\
\left(\text { mJy beam }^{-1}\right)\end{array}$ & $\begin{array}{l}\text { Total Mass } \\
\qquad\left(M_{\odot}\right)\end{array}$ & $\begin{array}{c}\theta_{\mathrm{dec}} \\
(\operatorname{arcsec})\end{array}$ & Axis Ratio & $\begin{array}{c}n_{1 e 4} \\
\left(\mathrm{~cm}^{-3}\right)\end{array}$ \\
\hline \multicolumn{8}{|c|}{ Perseus } \\
\hline Per-Bolo $5 \ldots \ldots \ldots \ldots$ & 032522.2 & +304509 & 727 & 2.55 & 47.3 & 1.8 & $6.5 \times 10^{5}$ \\
\hline Per-Bolo $8 \ldots \ldots \ldots \ldots . .$. & 032535.9 & +304517 & 2241 & 5.86 & 36.6 & 1.2 & $20.6 \times 10^{5}$ \\
\hline Per-Bolo $10 \ldots \ldots \ldots$. & 032538.4 & +304358 & 979 & 2.54 & 43.1 & 1.0 & $8.5 \times 10^{5}$ \\
\hline Per-Bolo $18 \ldots \ldots \ldots . .$. & 032636.9 & +301523 & 225 & 0.50 & 25.9 & 1.2 & $1.8 \times 10^{5}$ \\
\hline Per-Bolo 21 ........... & 032737.5 & +301353 & 164 & 0.34 & 39.1 & 1.4 & $1.5 \times 10^{5}$ \\
\hline Per-Bolo $22 \ldots \ldots \ldots \ldots$ & 032739.3 & +301253 & 317 & 0.51 & 35.8 & 1.2 & $2.9 \times 10^{5}$ \\
\hline Per-Bolo 23 ....................... & 032741.7 & +301224 & 283 & 0.50 & 45.2 & 1.2 & $2.8 \times 10^{5}$ \\
\hline Per-Bolo 24 ....................... & 032747.9 & +301202 & 220 & 0.82 & 54.9 & 1.5 & $2.3 \times 10^{5}$ \\
\hline Per-Bolo $25 . . . . . . . . . .$. & 032832.1 & +311109 & 106 & 0.29 & 70.9 & 2.6 & $0.8 \times 10^{5}$ \\
\hline
\end{tabular}

NoTES.- Units of right ascension are hours, minutes, and seconds, and units of declination are degrees, arcminutes, and arcseconds. Table columns are as for Table 1. Note that the Ophiuchus identifications are different than in Tables 1 and 2 of Paper II for Oph-Bolo 35 and above due to an adjustment of the source list, resulting in 43 rather than 44 sources. The peak flux, total mass, sizes, etc., for Oph-Bolo 35 and Oph-Bolo 36 are also different than in Paper II. Table 2 is published in its entirety in the electronic edition of the Astrophysical Journal. A portion is shown here for guidance regarding its form and content. 

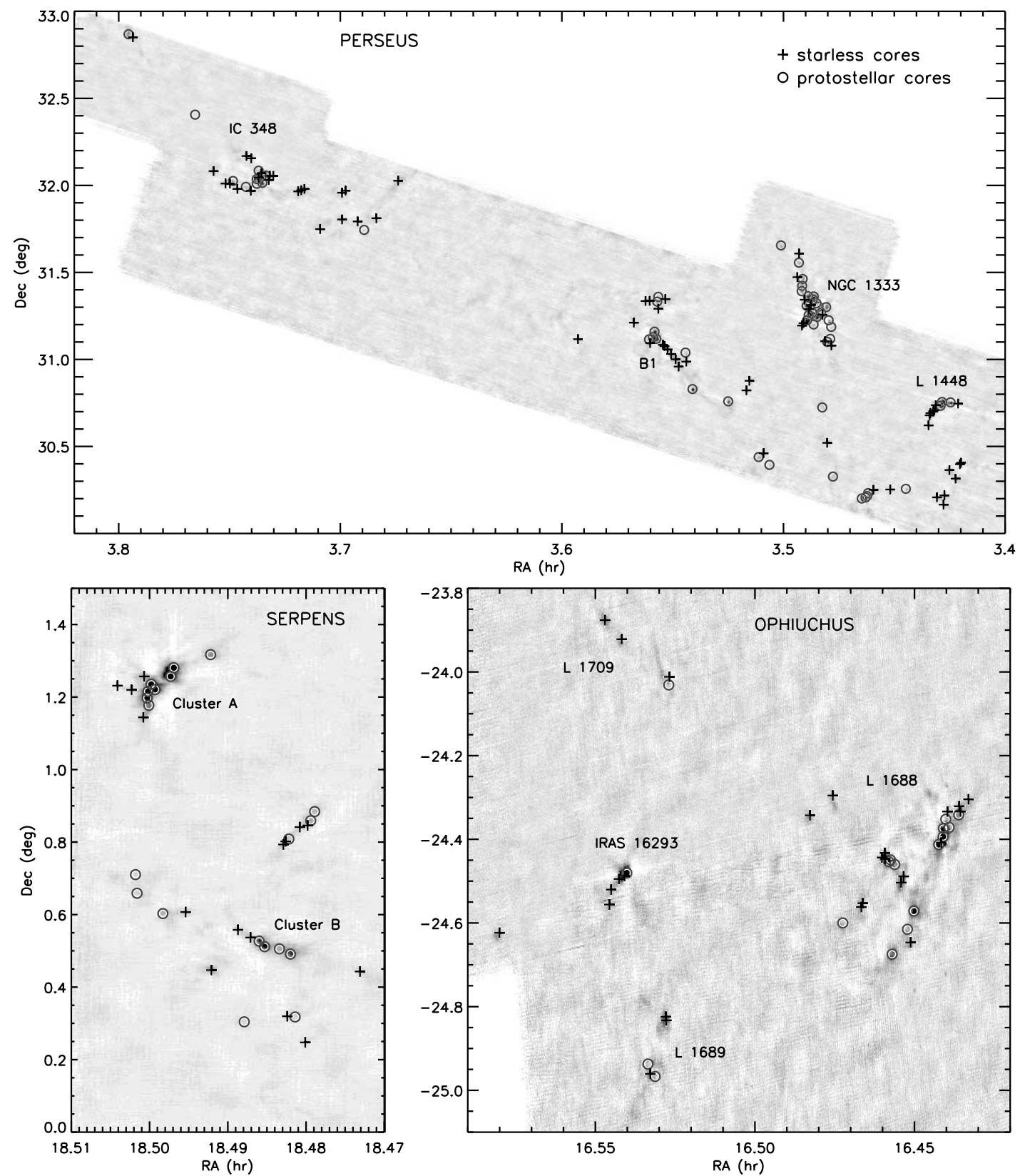

FIG. 3.-Bolocam maps of Perseus, Serpens, and Ophiuchus, with the positions of starless and protostellar cores indicated. Identified cores have peak flux densities of at least $5 \sigma$, where $\sigma$ is the local rms noise level (on average $\sigma=15 \mathrm{mJy} \mathrm{beam}^{-1}$ in Perseus, $10 \mathrm{mJy} \mathrm{beam}^{-1}$ in Serpens, and $25 \mathrm{mJy} \mathrm{beam}^{-1}$ in Ophiuchus). Note that due to the large scales, individual structures are difficult to see. Regions of the maps with no detected sources have been trimmed for this figure. Starless and protostellar cores cluster together throughout each cloud, with both populations tending to congregate along filamentary cloud structures. There are a few exceptional regions, however, which are dominated by either starless or protostellar cores (e.g., the B1 ridge, Serpens cluster A). [See the electronic edition of the Journal for a color version of this figure.]

level, and positions are determined using a surface-brightnessweighted centroid, as described in Paper III. The Bolocam maps of Perseus, Serpens, and Ophiuchus are shown in Figure 3, with the positions of starless and protostellar cores indicated. The average $1 \sigma \mathrm{rms}$ is $15 \mathrm{mJy}_{\text {beam }}^{-1}$ in Perseus, $10 \mathrm{mJy}^{\text {beam }}{ }^{-1}$ in Serpens, and 25 mJy beam $^{-1}$ in Ophiuchus.

General core statistics, including the number of starless $\left(N_{\mathrm{SL}}\right)$ and protostellar $\left(N_{\mathrm{PS}}\right)$ cores in each cloud, as well as the ratio $N_{\mathrm{SL}} / N_{\mathrm{PS}}$, are given in Table 3 . Note that the number of starless and protostellar cores are approximately equal in each cloud $\left(N_{\mathrm{SL}} / N_{\mathrm{PS}}=1.2\right.$ in Perseus, 0.8 in Serpens, and 1.4 in Ophiuchus), a fact that will be important for our discussion of the starless core lifetime in $\S 6$. The last column of Table 3 gives the number of individual $1.1 \mathrm{~mm}$ cores that are associated with more than one
TABLE 3

Statistics of $1.1 \mathrm{~mm}$ Cores in the Three Clouds

\begin{tabular}{crcccc}
\hline \hline Cloud & $N_{\text {total }}{ }^{\mathrm{a}}$ & $N_{\mathrm{SL}}{ }^{\mathrm{b}}$ & $N_{\mathrm{PS}}{ }^{\mathrm{c}}$ & $N_{\mathrm{SL}} / N_{\mathrm{PS}}$ & $N_{\mathrm{PS}}$ (Multiple) $^{\mathrm{d}}$ \\
\hline Perseus ................ & 122 & 67 & 55 & 1.2 & 13 \\
Serpens ............... & 35 & 15 & 20 & 0.8 & 11 \\
Ophiuchus ........... & 43 & 26 & 17 & 1.5 & 3 \\
\hline
\end{tabular}

${ }^{\text {a }}$ Total number of identified $1.1 \mathrm{~mm}$ cores.

b Number of starless $1.1 \mathrm{~mm}$ cores, i.e., cores that do not have a protostar candidate located within $1.0 \times \theta_{1 \mathrm{~mm}}$ of the core position.

${ }^{\mathrm{c}}$ Number of protostellar cores.

d Number of protostellar cores that are associated with more than one candidate cold protostar (each within $1.0 \times \theta_{1 \mathrm{~mm}}$ of the core position). 


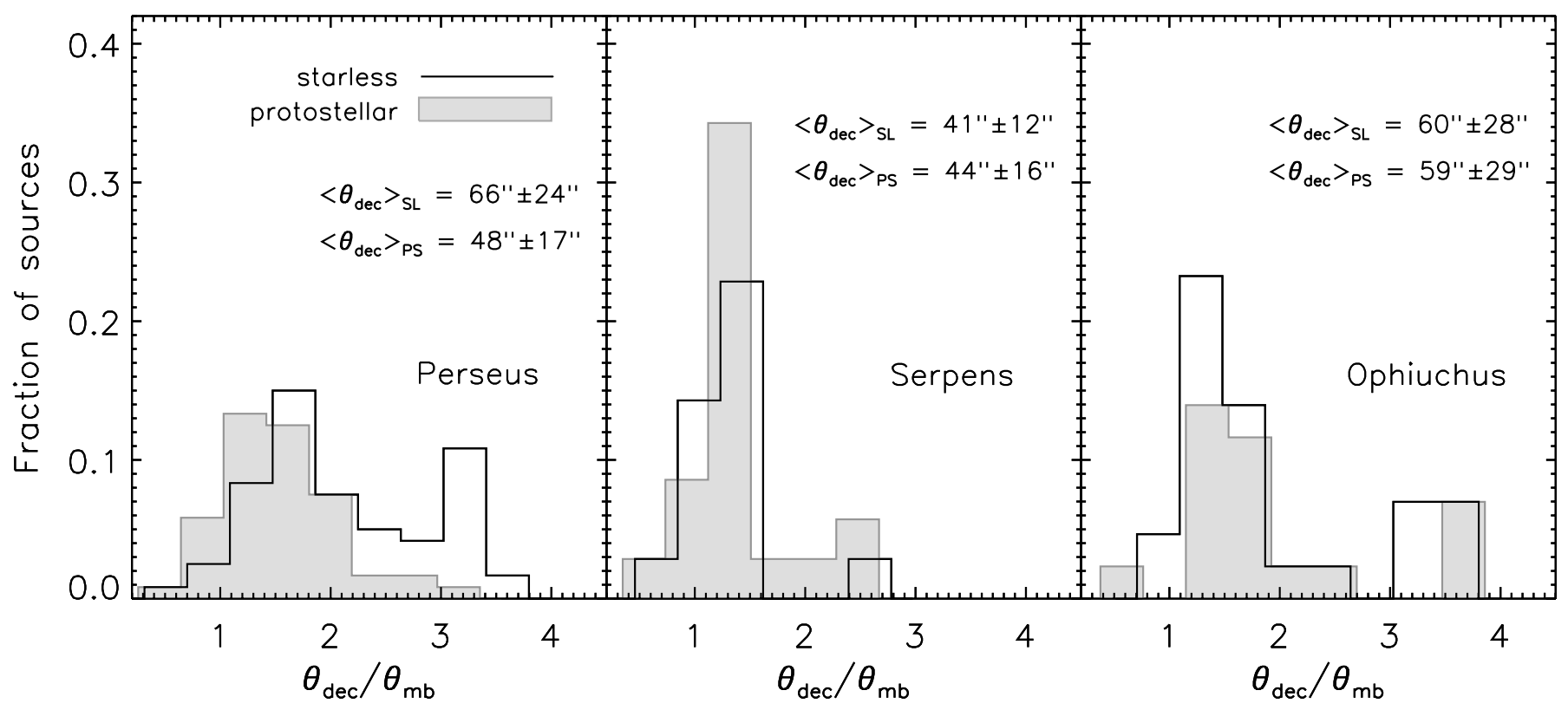

FIG. 4.-Distributions of the angular deconvolved sizes of starless and protostellar cores in the three clouds. The size is given in units of the beam FWHM $\left(\theta_{\mathrm{mb}}\right)$, and the mean of each distribution \pm the dispersion in the sample is listed. Starless cores are larger on average than protostellar cores in Perseus, with a flattened distribution out to $3.5 \theta_{\mathrm{mb}}$. In Serpens and Ophiuchus, however, there is little difference between the starless and protostellar distributions, and there are fewer very large cores, particularly in Serpens. The value of $\theta_{\mathrm{dec}} / \theta_{\mathrm{mb}}$ can be used to infer the steepness of the source radial density profile (see text and Young et al. 2003).

candidate protostar (each located within $1 \times \theta_{1 \mathrm{~mm}}$ of the core position). There are 13 such "multiple" protostellar sources in Perseus (24\% of the protostellar core sample), 11 in Serpens (55\%), and 3 in Ophiuchus (17\%). In general there are two or three candidate protostars associated with each "multiple" core, with the exception of one core in Serpens (5 candidate protostars). Throughout this work multiple protostellar cores are treated as single objects.

We now compare the physical properties of the starless and protostellar core populations in each cloud, with two primary goals. Isolating a starless sample allows us to probe the initial conditions of star formation, and differences between the starless and protostellar core samples are indicative of how the formation of a central protostar alters core properties. In the following sections we follow the methodology of Paper III, examining the sizes and shapes of cores, their peak and mean densities, distributions of core mass versus size, spatial clustering properties, and relationship to the surrounding cloud column density.

\subsection{Sizes and Shapes}

Source angular FWHM sizes $\left(\theta_{\text {meas }}\right)$ are measured by fitting an elliptical Gaussian after masking out nearby sources using a mask radius equal to half the distance to the nearest neighbor (see Paper II). The angular deconvolved core size is the geometric mean of the deconvolved minor and major angular FWHM sizes: $\theta_{\text {dec }}=\left(\theta_{d, \text { maj }} \theta_{d, \text { min }}\right)^{1 / 2}$, where $\theta_{d}=\left(\theta_{\text {meas }}^{2}-\theta_{\mathrm{mb}}^{2}\right)^{1 / 2}$ and $\theta_{\mathrm{mb}}=$ $31^{\prime \prime}$ is the beam FWHM. Deconvolved sizes for starless and protostellar cores are given in Tables 1 and 2, respectively, and the size distributions are plotted in Figure 4.

As discussed in Paper III, the measured size does not necessarily represent a physical boundary, but rather is a characteristic scale that depends on the linear resolution and intrinsic source density profile. For sources with power-law density profiles, which do not have a well-defined size, $\theta_{\mathrm{dec}} / \theta_{\mathrm{mb}}$ is independent of distance and simply related to the index of the power law (Young et al. 2003). According to the correlation between $\theta_{\mathrm{dec}} / \theta_{\mathrm{mb}}$ and density-power-law exponent $p$ found by Young et al. (2003) a mean $\theta_{\mathrm{dec}} / \theta_{\mathrm{mb}}$ value of $50^{\prime \prime} / 31^{\prime \prime}=1.6$ for protostellar cores in Perseus implies an average power-law index of $p \sim 1.4-1.5$. Many well-known protostellar sources have been found to have envelopes consistent with power-law density profiles, as determined by high-resolution imaging combined with radiative transfer modeling. Our inferred average index for Perseus protostellar cores $(p=1.4-1.5)$ is consistent with the mean $p \sim 1.6$ from radiative transfer modeling of Class 0 and Class I envelopes (Shirley et al. 2002; Young et al. 2003). For reference, a singular isothermal sphere (SIS) has $p=2\left(\theta_{\mathrm{dec}} / \theta_{\mathrm{mb}} \sim 0.9\right)$, and the profile expected for a free-falling envelope is $p=1.5\left(\theta_{\mathrm{dec}} / \theta_{\mathrm{mb}} \sim\right.$ 1.6) (Shu 1977; Young et al. 2003).

Starless cores in Perseus are larger on average than protostellar cores (Fig. 4); the starless distribution is relatively flat, with a few barely resolved cores, and several larger than $3 \theta_{\mathrm{mb}}$. The Student's $t$-test confirms that the mean sizes of starless and protostellar cores are significantly different (significance of the $t$-statistic is $2 \times 10^{-5}$ ). A mean $\theta_{\mathrm{dec}} / \theta_{\mathrm{mb}}$ of 2.2 for starless cores in Perseus would imply an extremely shallow mean power-law index of $p \sim 1.1$, and the maximum value $\left(\theta_{\mathrm{dec}} / \theta_{\mathrm{mb}} \sim 3.5\right)$ would correspond to $p<0.8$. Other flattened profiles, such as the Bonnor-Ebert (BE) sphere (Ebert 1955; Bonnor 1956), could also produce large $\theta_{\mathrm{dec}} / \theta_{\mathrm{mb}}$ values. A BE profile with a central density of $10^{5} \mathrm{~cm}^{-3}$ and an outer radius of $6 \times 10^{4} \mathrm{AU}$ would correspond to $\theta_{\mathrm{dec}} / \theta_{\mathrm{mb}}=2.0$ at the distance of Perseus. There is significant observational evidence that many starless cores do indeed look like BE spheres (e.g., Johnstone et al. 2000; Shirley et al. 2000; Alves et al. 2001; Evans et al. 2001). We conclude that very large starless cores are more consistent with $\mathrm{BE}$ spheres or other flattened density profiles $(p \lesssim 1)$ than with the classical precollapse SIS $(p=2){ }^{9}$

\footnotetext{
${ }^{9}$ As the pressure-truncated boundary radius of the BE solution has not been observationally verified, the resemblance between starless cores and BE profiles is necessarily limited to the region inside the boundary radius. Also note that a sufficiently centrally condensed BE sphere will be indistinguishable from a power law on the scales to which we are sensitive.
} 


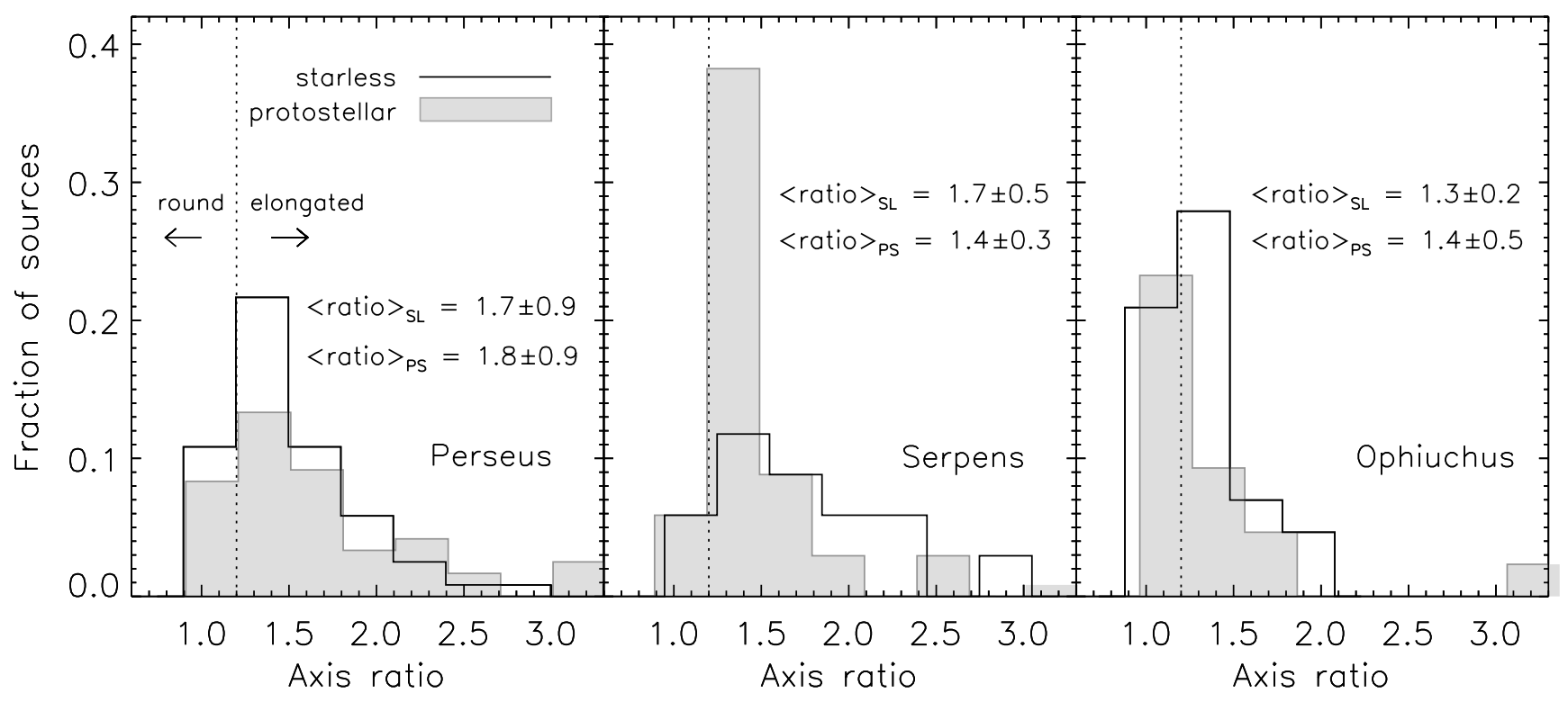

FIG. 5.-Distributions of the axis ratios of starless and protostellar cores. Cores with axis ratios $<1.2$ are considered round and those with axis ratios $>1.2$ elongated, based on Monte Carlo simulations (Paper I). Only in Serpens, where starless cores tend to be slightly more elongated than protostellar cores, is there a distinguishable difference between the starless and protostellar populations.

Despite their size differences, there is little difference in the axis ratios of starless and protostellar cores in Perseus (Fig. 5). Both populations are slightly elongated on average. The axis ratio is defined at the half-maximum contour, using deconvolved sizes: $\theta_{d, \text { maj }} / \theta_{d, \min }$. Values for individual cores are given in Tables 1 and 2. Starless and protostellar cores have mean axis ratios of $1.7 \pm 0.9$ and $1.8 \pm 0.9$. Standard deviations quoted here and in the rest of $\S 3$ are dispersions in the sample, not errors in the mean. Monte Carlo tests (Paper I) indicate that cores with axis ratios less than 1.2 should be considered round.

In contrast to Perseus, starless cores in Serpens are no larger than protostellar cores. Mean values for both $\left(\theta_{\mathrm{dec}} / \theta_{\mathrm{mb}} \sim 1.5\right)$ correspond to an average power-law index of $p=1.5$, similar to that found for protostellar cores in Perseus, and to typical radiative transfer modeling results for protostellar envelopes. Starless cores in Serpens may be more elongated than protostellar cores (mean axis ratios of $1.7 \pm 0.5$ and $1.4 \pm 0.3$, respectively), but the difference is not statistically significant.

In Ophiuchus there is no measurable difference between the starless and protostellar populations. The starless and protostellar samples have similar mean sizes, and both display a bimodal behavior (Fig. 4). The lower peak is similar to the single peak seen in Serpens at $\theta_{\mathrm{dec}}=(1-2) \theta_{\mathrm{mb}}$, while the smaller upper peak is at sizes of $\theta_{\mathrm{dec}}=(3-4) \theta_{\mathrm{mb}}$, comparable to the largest starless cores in Perseus. The mean $\theta_{\mathrm{dec}} / \theta_{\mathrm{mb}}$ for protostellar cores (1.9) corresponds to an average power-law index of $p=1.3$. Both starless and protostellar cores in Ophiuchus appear fairly round, with the mean axis ratios of $1.3 \pm 0.2$ and $1.4 \pm 0.5$, respectively (Fig. 5). As discussed in Paper III, larger axis ratios in Perseus and Serpens may be at least partly an effect of the lower linear resolution in those clouds compared to Ophiuchus (e.g., blending).

Were we to calculate linear sizes, cores in Ophiuchus would appear smaller by nearly a factor of 2 compared to Perseus and Serpens, given the smaller distance to Ophiuchus. This is primarily a systematic effect of the linear resolution, however. In Paper III we found that convolving the Ophiuchus map with a larger beam to match the linear resolution of Perseus and Serpens produced larger linear core sizes by nearly a factor of 2 , but similar measured $\theta_{\mathrm{dec}} / \theta_{\mathrm{mb}}$ values. Thus we focus here on angular sizes only.

To summarize, protostellar cores in all three clouds have mean sizes consistent with power-law density profiles with an average index $p=1.3-1.5$. Starless cores in Perseus are significantly larger on average than protostellar cores, suggestive of BE spheres or other shallow density profiles. Starless cores are quite compact in Serpens, while both starless and protostellar cores in Ophiuchus display a bimodal distribution of sizes, with a few very large cores. The deficit of cores with $\theta_{\mathrm{dec}} / \theta_{\mathrm{mb}}>2$ in Serpens and Ophiuchus as compared to Perseus may be related to the general lack of isolated sources in those clouds; the measured size of a core is limited by the distance to the nearest neighboring source, so sizes will tend to be smaller in crowded regions (e.g., L1688 Ophiuchus) than for isolated sources.

\subsection{Core Masses and Densities}

Core total masses, given in Tables 1 and 2, are calculated from the total $1.1 \mathrm{~mm}$ flux, $S_{1.1 \mathrm{~mm}}$ :

$$
M=\frac{d^{2} S_{\nu}}{B_{\nu}\left(T_{D}\right) \kappa_{\nu}},
$$

where $d$ is the cloud distance, $B_{\nu}$ is the Planck function at dust temperature $T_{D}$, and $\kappa_{1.1 \mathrm{~mm}}=0.0114 \mathrm{~cm}^{2} \mathrm{~g}^{-1}$ is the dust opacity. The total $1.1 \mathrm{~mm}$ flux density is integrated in the largest aperture $\left(30^{\prime \prime}-120^{\prime \prime}\right.$ diameters in steps of $\left.10^{\prime \prime}\right)$ that is smaller than the distance to the nearest neighboring source. We assume that the dust emission at $\lambda=1.1 \mathrm{~mm}$ is optically thin, and that $T_{D}$ and $\kappa_{1.1 \mathrm{~mm}}$ are independent of position within a core. The value of $\kappa_{1.1 \mathrm{~mm}}$ is interpolated from Table 1, column (5), of Ossenkopf \& Henning (1994) for dust grains with thin ice mantles, and includes a gas-to-dust mass ratio of 100 . A recent measurement of $\kappa$ based on near-IR data and 450 and $850 \mu \mathrm{m}$ SCUBA maps yields $\kappa_{1.1 \mathrm{~mm}}=0.0088 \mathrm{~cm}^{2} \mathrm{~g}^{-1}$ (Shirley et al. 2007), which would increase our masses by a factor of 1.3.

The value of $T_{D}$ should depend on whether a core is starless or has an internal source of luminosity, so we assume a slightly 


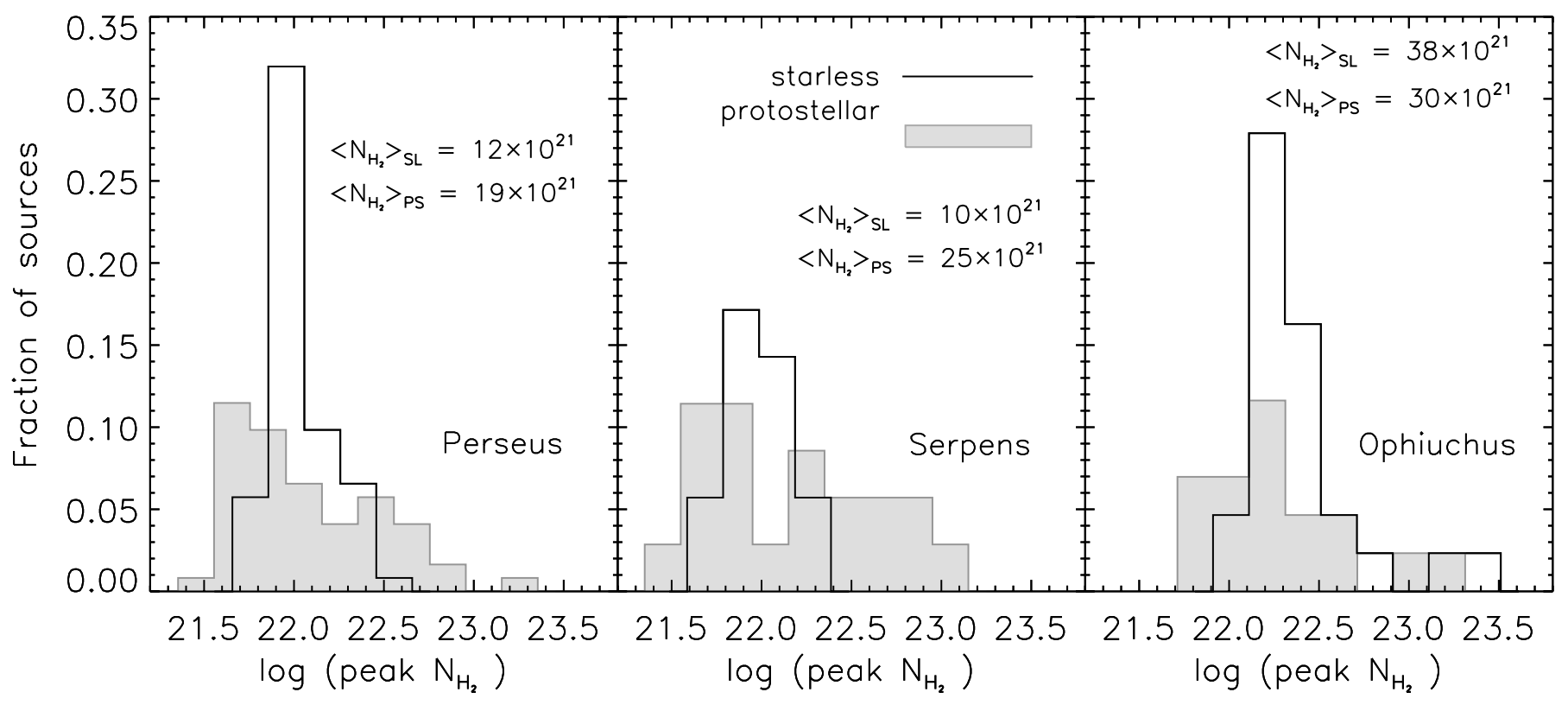

Fig. 6.-Distributions of the peak column density $N_{\mathrm{H}_{2}}$ of starless and protostellar cores. The peak $N_{\mathrm{H}_{2}}$ values of starless cores are considerably lower than those of protostellar cores in both Perseus and Serpens, by factors of approximately 1.6 and 2.5, respectively. In Ophiuchus there is no significant difference in the mean values, but in all three clouds the starless distribution is confined to a narrowly peaked distribution.

higher temperature for protostellar cores $\left(T_{D}=15 \mathrm{~K}\right)$ than for starless cores $\left(T_{D}=10 \mathrm{~K}\right)$. For dense regions without internal heating, the mean temperature is about $10 \mathrm{~K}$, warmer on the outside and colder on the inside (Evans et al. 2001). A recent $\mathrm{NH}_{3}$ survey of the Bolocam cores in Perseus confirms that the median kinetic temperature of starless cores in Perseus is $10.8 \mathrm{~K}$ (see S. Schnee et al. 2008, in preparation, and $\S 4$ ). Our assumed value of $15 \mathrm{~K}$ for protostellar cores is the average isothermal dust temperature found from radiative transfer models of Class 0 and Class I protostars (Shirley et al. 2002; Young et al. 2003). The isothermal dust temperature is the temperature that, when used in an isothermal mass equation (e.g., eq. [1]), yields the same mass as a detailed radiative transfer model including temperature gradients. There is a factor of 1.9 difference in mass between assuming $T_{D}=10$ and $15 \mathrm{~K}$.

Figures 6 and 7 compare the peak and mean densities of starless and protostellar cores in each cloud. We use the peak column density $N_{\mathrm{H}_{2}}$, calculated from the peak $1.1 \mathrm{~mm}$ flux density $S_{1.1 \mathrm{~mm}}^{\text {beam }}$, as a measure of the peak density:

$$
N\left(\mathrm{H}_{2}\right)=\frac{S_{\nu}^{\text {beam }}}{\Omega_{\text {beam }} \mu_{\mathrm{H}_{2}} m_{\mathrm{H}} \kappa_{\nu} B_{\nu}\left(T_{D}\right)},
$$

with $N\left(\mathrm{H}_{2}\right) / A_{V}=0.94 \times 10^{21} \mathrm{~cm}^{-2} \mathrm{mag}^{-1}$ (Frerking et al. 1982). Here $\Omega_{\text {beam }}$ is the beam solid angle, $m_{\mathrm{H}}$ is the mass of hydrogen, and $\mu_{\mathrm{H}_{2}}=2.8$ is the mean molecular weight per $\mathrm{H}_{2}$ molecule. As discussed in Paper III, the $1.1 \mathrm{~mm}$ emission detected by Bolocam traces significantly higher column densities than other tracers such as the reddening of background stars.

Mean particle densities, given in Tables 1 and 2, are calculated within a fixed linear aperture of diameter $10^{4} \mathrm{AU}$ :

$$
n_{1 e 4}=\frac{3 M_{1 e 4}}{4 \pi\left(D_{1 e 4} / 2\right)^{3} \mu m_{\mathrm{H}}},
$$

where $D_{1 e 4}$ is the aperture size $\left(10^{4} \mathrm{AU}\right), M_{1 e 4}$ is the mass calculated from the $1.1 \mathrm{~mm}$ flux within that aperture, and $\mu=$ 2.33 is the mean molecular weight per particle. An aperture of
$10^{4} \mathrm{AU}$ corresponds to approximately $40^{\prime \prime}$ in Perseus and Serpens, and $80^{\prime \prime}$ in Ophiuchus. Note that $n_{1 e 4}$ does not depend on the core size; while in some cases $10^{4} \mathrm{AU}$ may be considerably smaller or larger than the source FWHM, a fixed linear aperture is used here to mitigate the effects of linear resolution, which was found in Paper III to significantly bias the mean density calculated within the FWHM contour.

The average peak column density $\left(N_{\mathrm{H}_{2}}\right)$ for starless cores in Perseus is $12 \times 10^{21} \mathrm{~cm}^{-2}$, while the average for protostellar cores is $50 \%$ higher, $19 \times 10^{21} \mathrm{~cm}^{-2}$, with a much wider dispersion (the significance of the Student's $t$-statistic is 0.03). Similarly, the typical mean density $\left(n_{1 e 4}\right)$ of starless cores $(1.7 \times$ $\left.10^{5} \mathrm{~cm}^{-3}\right)$ is a factor of 3 smaller than that of protostellar cores $\left(5.1 \times 10^{5} \mathrm{~cm}^{-3}\right.$; Student's $t$-test significance $\left.6 \times 10^{-5}\right)$. The large difference in mean densities is due primarily to the significantly smaller sizes of protostellar cores in Perseus. Recently, Jørgensen et al. (2007) found a similar result for Perseus, comparing SCUBA $850 \mu \mathrm{m}$ cores with and without internal luminosity sources, as determined using Spitzer c2d data. Those authors concluded that cores with embedded YSOs (located within $15^{\prime \prime}$ of the core position) have higher "concentrations" on average. Note that average $n_{1 e 4}$ values are much larger than the minimum detectable density of $\sim 2 \times 10^{4} \mathrm{~cm}^{-3}$ from Paper III, due in part to few sources near the detection limit and in part to the difference in calculating densities in a fixed aperture.

Density distributions for starless and protostellar cores in Serpens are similar to those in Perseus. Peak $N_{\mathrm{H}_{2}}$ values are substantially smaller for starless $\left(\left\langle N_{\mathrm{H}_{2}}\right\rangle=10 \times 10^{21} \mathrm{~cm}^{-2}\right)$ than for protostellar $\left(\left\langle N_{\mathrm{H}_{2}}\right\rangle=25 \times 10^{21} \mathrm{~cm}^{-2}\right.$; Student's $t$-test significance 0.04$)$ cores, and form a much narrower distribution. Likewise, typical mean densities of starless cores $\left(1.4 \times 10^{5} \mathrm{~cm}^{-3}\right)$ are nearly 3 times smaller than those of protostellar cores $(3.5 \times$ $10^{5} \mathrm{~cm}^{-3}$; Student's $t$-test significance 0.03 ). In contrast to Perseus, however, mean density differences in Serpens are due entirely to the higher masses of protostellar cores, as starless and protostellar cores have similar sizes in Serpens. As was the case for core sizes and shapes, there is essentially no difference between the peak or mean densities of starless and protostellar cores in 


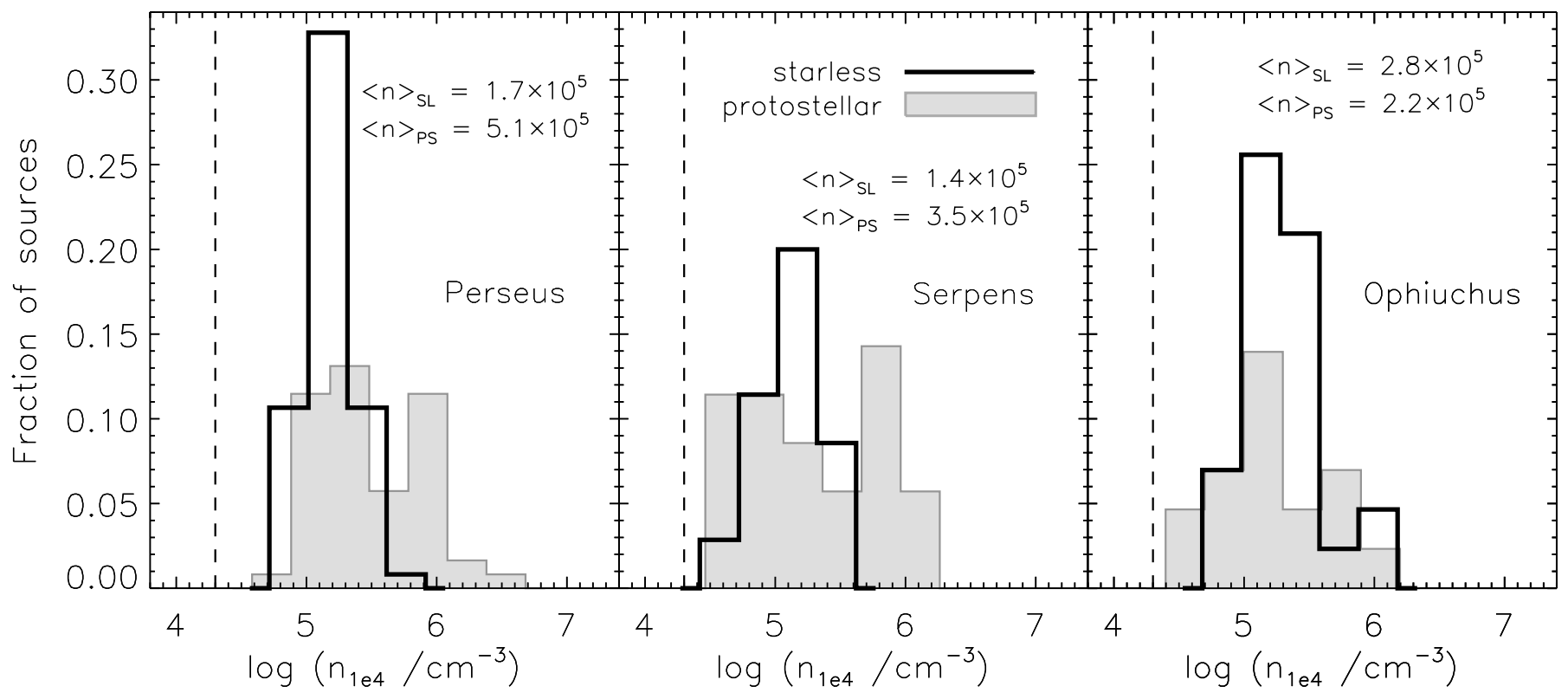

FIG. 7.-Distributions of the mean density $n_{1 e 4}$ of starless and protostellar cores, where the density is calculated in a fixed linear aperture of diameter $10^{4}$ AU ( $\left.\S 3.2\right)$. As for the peak $N_{\mathrm{H}_{2}}$ distribution (Fig. 6), starless cores tend to have lower mean densities than protostellar cores in Perseus and Serpens, by approximately a factor of 3. In Ophiuchus, by contrast, there is almost no difference between the two distributions. Note that the Bolocam surveys are only sensitive to relatively dense cores with $n \gtrsim 2 \times 10^{4} \mathrm{~cm}{ }^{-3}$ (dashed lines; see Paper III).

Ophiuchus. Average peak $N_{\mathrm{H}_{2}}$ values are similar [(30-40) $\times$ $\left.10^{21} \mathrm{~cm}^{-2}\right]$ and the two distributions have similar dispersions, and average $n_{1 e 4}$ values are $(2.2-2.8) \times 10^{5} \mathrm{~cm}^{-3}$.

To summarize, peak column densities and mean densities of starless cores in Perseus and Serpens are lower on average than for protostellar cores, whereas in Ophiuchus there is no significant difference between the starless and protostellar populations. The $N_{\mathrm{H}_{2}}$ distributions for starless cores are quite narrow in all three clouds, indicating a small range of column densities. This narrow distribution more likely represents an upper limit to $N_{\mathrm{H}_{2}}$ for starless cores, as we are not sensitive to very large low-mass cores (see $\S 3.3$ ), but in general cores with the highest column densities tend to be protostellar.

\subsection{The Mass-versus-Size Distribution}

Figure 8 plots total core mass versus angular FWHM size for starless and protostellar cores in Perseus. The beam size and empirical $50 \%$ and $90 \%$ completeness limits as a function of size, derived from Monte Carlo simulations (Paper I), are indicated. Completeness limits are lower for protostellar cores by a factor of 2 due to the higher dust temperature assumed in the mass calculation $(15 \mathrm{~K})$ compared to starless cores $(10 \mathrm{~K})$. There is approximately a factor of 1.2 increase in mass between the $50 \%$ and $90 \%$ completeness curves, which holds true for the other clouds as well.

In this diagram, starless cores seem to follow a constant-surfacedensity $\left(M \propto R^{2}\right)$ curve, consistent with the narrow distribution of peak column densities (Fig. 6). Protostellar cores, in contrast, have a narrower range of sizes for a somewhat larger range of masses. This is a restatement of the results from $\S \S 3.1$ and 3.2: protostellar cores in Perseus are smaller and have higher mean densities than starless cores. Note that because our completeness limits are similar to $M \propto R^{2}$, the distribution of starless cores in mass and size only implies that the upper envelope of cores follows a constant-surface-density curve. For example, there could be a population of large, low-mass cores that we are unable to detect. Nevertheless, the two populations seem to fill different regions of the mass-versus-size parameter space.
Examining Figure 8, it is easy to imagine how protostellar cores in Perseus might have evolved from the current population of starless cores, by decreasing in size and increasing in density for a constant mass, until collapse and protostellar formation is triggered. Equivalently, the formation of a central protostar within a previously starless core is associated with a decrease in core size and an increase in core density.

Such a simple scenario is not consistent with the other clouds, however, as is evident in Figure 9. Although protostellar cores in Serpens have a small range in sizes for a large range of masses, as seen in Perseus, there is no population of large starless cores in Serpens. In fact, it is unclear how the relatively massive protostellar cores $\left(\langle M\rangle_{\mathrm{PS}}=2.1\right)$ in Serpens could have evolved from the current population of compact, low-mass starless cores $\left(\langle M\rangle_{\mathrm{SL}}=0.9\right)$. It appears that Serpens has exhausted its reserve of starless cores with $M \gtrsim 2 M_{\odot}$; unless new cores are formed from the lower density medium, future star formation in the cloud will result in stars of considerably lower mass than the current protostellar population.

This deficit of starless cores at higher core masses may be the result of a mass dependence in the timescale for protostellar formation, with higher mass cores forming protostars more quickly (Hatchell \& Fuller 2008). In both Serpens and Perseus the most massive cores tend to be protostellar in nature, although the trend is much more extreme in Serpens. In Ophiuchus, where core masses are lower on average than in the other clouds, no such distinction between starless and protostellar cores is seen. There is essentially no difference between the starless and protostellar populations in Ophiuchus, suggesting very little core evolution after the formation of a protostar.

\subsection{Relationship to Cloud Column Density}

We use the cumulative fraction of starless and protostellar cores as a function of cloud $A_{V}$, shown in Figure 10, to quantify the relationship between dense cores and the surrounding cloud material. Visual extinction $\left(A_{V}\right)$ is a measure of the cloud column density, and is derived based on the reddening of background 


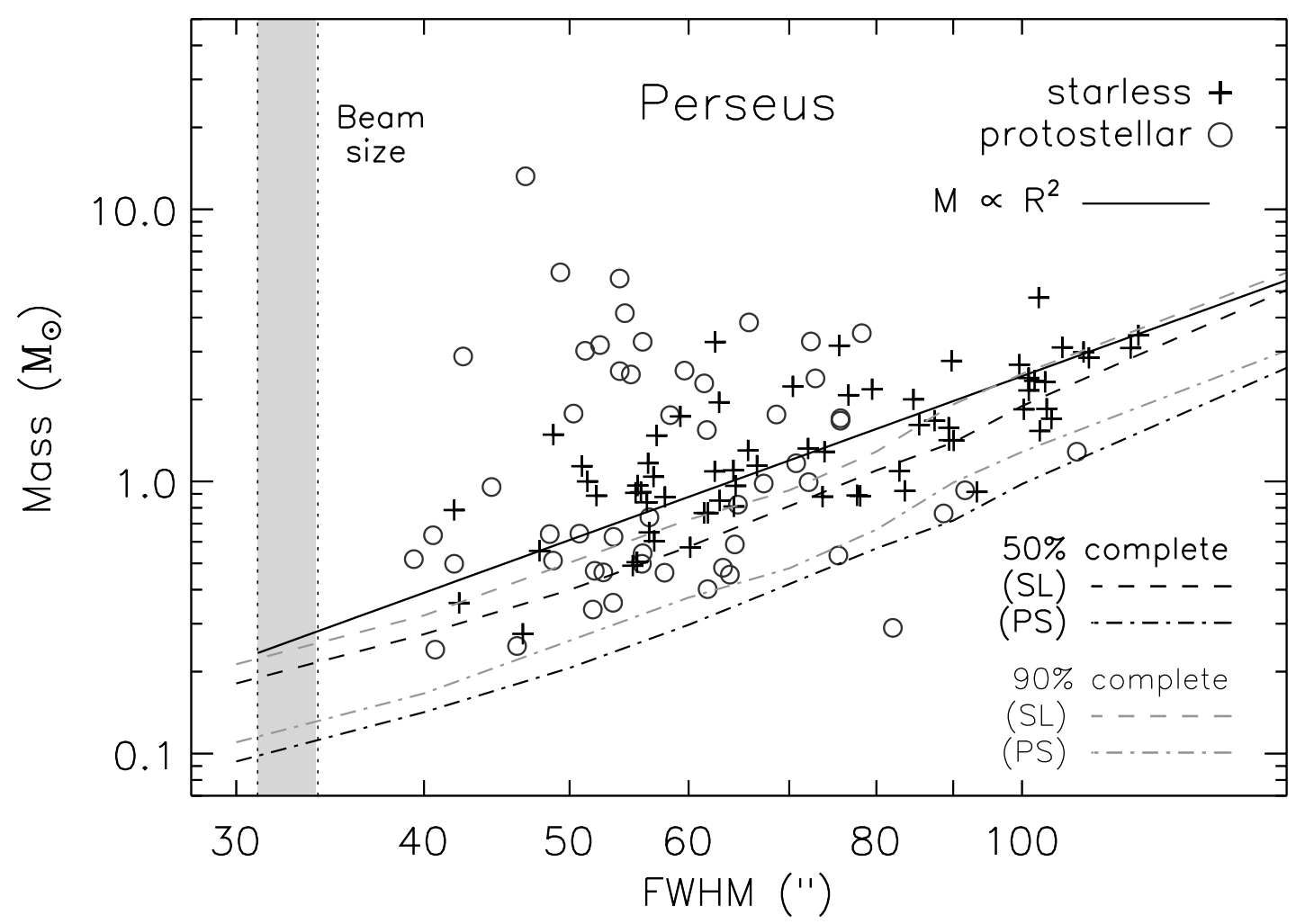

FIG. 8. - Total mass vs. angular FWHM size for starless and protostellar cores in Perseus. Dashed and dash-dotted lines indicate empirically derived 50\% (and 90\%; light gray) completeness limits for starless and protostellar cores, respectively. The two populations seem to inhabit different regions of the parameter space: starless cores tend to follow a constant-surface-density relationship $\left(M \propto R^{2}\right.$; solid line), consistent with their narrow distribution in column density (Fig. 6), while protostellar cores have a wide range in masses for a relatively small range in sizes. This relationship suggests a simple explanation for how the protostellar cores might have evolved from a similar population of starless cores, with individual cores becoming smaller and denser at a constant mass until protostar formation is triggered. [See the electronic edition of the Journal for a color version of this figure.]

2MASS and IRAC sources, as described in Paper III and Huard et al. (2006). In Paper III, we found that $75 \%$ of $1.1 \mathrm{~mm}$ cores in Perseus, Serpens, and Ophiuchus are found at visual extinctions of $A_{V} \gtrsim 8,15$, and 20-23 mag, respectively. Although these values do not define a strict threshold, below these $A_{V}$ levels the likelihood of finding a $1.1 \mathrm{~mm}$ core is very low. Here we investigate whether the relationship between dense cores and cloud column density is different for starless and protostellar cores.

Figure 10 demonstrates that both starless and protostellar cores are found primarily at high cloud column densities $\left(A_{V}>6 \mathrm{mag}\right)$ :

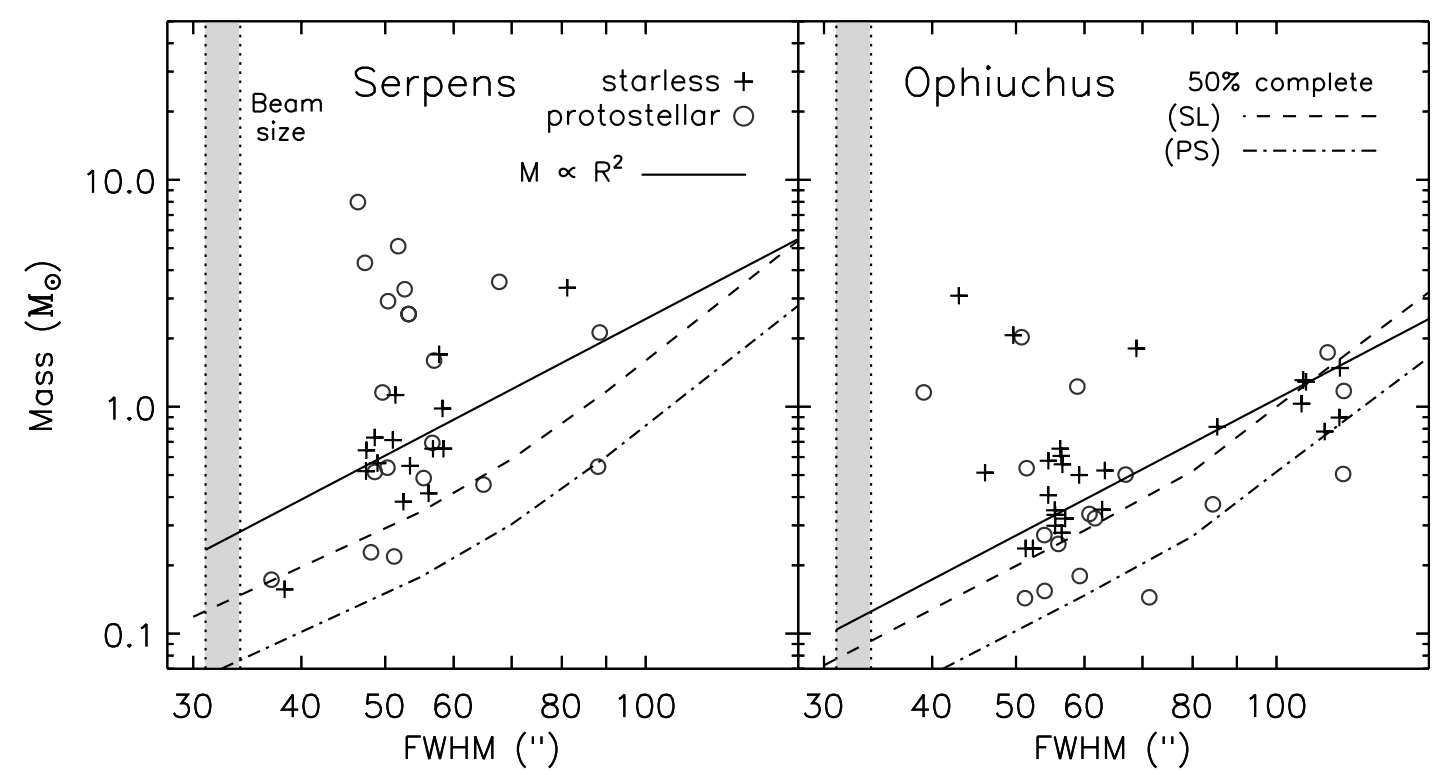

FIG. 9.- Total mass vs. angular FWHM size for starless and protostellar cores in Serpens and Ophiuchus. Lines are as in Fig. 8; as for Perseus, the 90\% completeness curves are approximately a factor of 1.2 higher in mass than the $50 \%$ completeness curves, although they are omitted here for clarity. Unlike in Perseus, starless cores do not necessarily follow a constant-surface-density $\left(M \propto R^{2}\right)$ line. Strikingly, there is no population of large starless cores in Serpens; it is not clear how the current population of relatively massive protostellar cores could have evolved from such compact, low-mass starless cores. This discrepancy suggests that future star formation in the cloud may result in stars of considerably lower mass than the currently forming protostars. [See the electronic edition of the Journal for a color version of this figure.] 


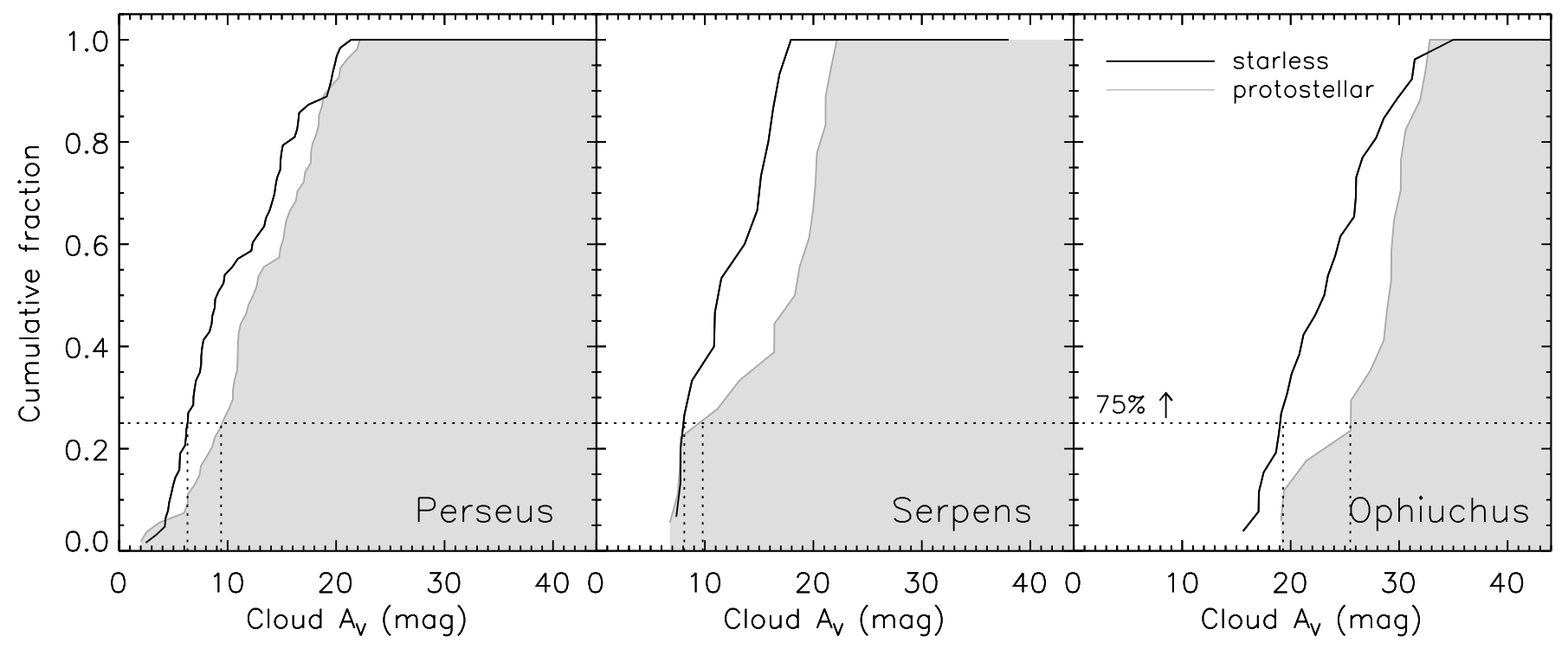

FIG. 10.-Cumulative fraction of starless and protostellar cores as a function of cloud $A_{V}$, where the $A_{V}$ is derived from the reddening of background stars in $2 \mathrm{MASS}$ and

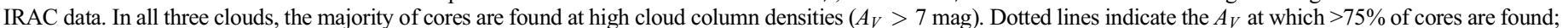

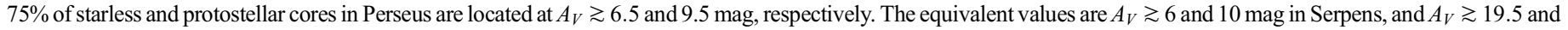
25.5 mag in Ophiuchus. There appears to be a strict extinction threshold in Serpens and Ophiuchus, with no cores found below $A_{V} \sim 7$ and 15 mag, respectively.

$75 \%$ of starless and protostellar cores in Perseus are located at $A_{V} \gtrsim 6.5$ and $9.5 \mathrm{mag}$, respectively, $A_{V} \gtrsim 8$ and $10 \mathrm{mag}$ in Serpens, and $A_{V} \gtrsim 19.5$ and $25.5 \mathrm{mag}$ in Ophiuchus. Note that these "threshold" values are significantly higher than the minimum cloud $A_{V}$, which is approximately 2 mag in Perseus and Ophiuchus, and $6 \mathrm{mag}$ in Serpens. Starless cores tend to be found at somewhat lower $A_{V}$ than protostellar cores; a two-sided K-S test yields probabilities of approximately $1 \%$ that the starless and protostellar distributions are drawn from the same parent distribution in each cloud. In Serpens and Ophiuchus there appear to be strict extinction thresholds for $1.1 \mathrm{~mm}$ cores at 7 and $15 \mathrm{mag}$, respectively. A few cores in Perseus and Serpens lie outside the $A_{V}$ map area, and may be associated with low- $A_{V}$ material.

As discussed in Paper III, an extinction threshold has been predicted by McKee (1989) for photoionization-regulated star formation in magnetically supported clouds. In this model, core collapse and star formation will occur only in shielded regions of a molecular cloud where $A_{V} \gtrsim 4-8 \mathrm{mag}$. The fact that $75 \%$ of both protostellar and starless cores are found above $A_{V} \sim 6$ mag in each cloud is consistent with this model; while it is certainly not the only explanation, magnetic fields may play a role in inhibiting collapse of cores, at least in the low column density regions of molecular clouds.

\subsection{Clustering}

Finally, we look at the spatial clustering of starless and protostellar cores. The spatial distributions of starless and protostellar cores in each cloud are shown in Figure 3. Starless and protostellar cores appear to cluster together for the most part, often congregating along filamentary structures in the clouds. Both populations occur in the known clusters (e.g., NGC 1333, L1688) and in a more distributed way across the clouds, with a few exceptions, such as in Perseus where there is a group of primarily starless cores (B1 ridge).

As a more quantitative measure of clustering, we use the twopoint correlation function (Fig. 11):

$$
w(r)=\frac{H_{s}(r)}{H_{r}(r)}-1,
$$

where $H_{s}(r)$ is the number of core pairs with separation between $\log (r)$ and $\log (r+d r)$, and $H_{r}(r)$ is similar but for a random distribution. The correlation function $w(r)$ is a measure of the excess clustering as compared to a random distribution of sources. The top panels of Figure 11 plot $w(r)$ as a function of source separation $r$, with the linear beam size and average source size indicated. The bottom panels plot $\log (w)$, with a power-law fit $\left[w(r) \propto r^{p}\right]$ for $r$ larger than the average source size. In essence, the amplitude of $w(r)$ is a measure of the magnitude of clustering, while the slope is a measure of how quickly clustering falls off on increasing scales.

The amplitude of $w(r)$ is higher for the protostellar samples in all three clouds, indicating that the degree of clustering is stronger on all spatial scales for protostellar cores. Visually, however, starless and protostellar cores tend to cluster in a similar way (Fig. 3), and this observation is supported by the similarity in the slope of $w(r)$ for starless and protostellar cores. In Serpens, $p=-1.4 \pm 0.3$ and $-1.3 \pm 0.6$ for the starless and protostellar populations, respectively. The Ophiuchus curves are quite noisy, but the best-fit slopes $(-1.3 \pm 0.3$ and $-2.2 \pm 0.4)$ are consistent within $2 \sigma$. For Perseus the starless correlation function is shallower by $4 \sigma(p=-1.14 \pm 0.13$ and $-1.5 \pm 0.1)$. A shallower slope suggests that, while the amplitude of clustering is weaker for starless cores, it does not fall off as fast at larger spatial scales.

A lower amplitude of clustering for starless cores as compared to protostellar cores in Perseus and Serpens is confirmed by the peak number of cores per square parsec: 10 and $16 \mathrm{pc}^{-2}$, respectively, for starless and protostellar cores in Perseus, and 4 and $8 \mathrm{pc}^{-2}$ in Serpens. These numbers suggest that clustering in the protostellar samples is a factor of 1.5-2 times stronger than in the starless samples. In Ophiuchus, however, the values of the starless and protostellar populations are identical $\left(12 \mathrm{pc}^{-2}\right)$.

There are at least three plausible reasons that clustering might be stronger for protostellar cores, two environmental and one evolutionary. The difference may be an environmental effect if cores that are located in regions of the cloud with higher gas density are more likely to collapse to form protostars. In that case, more clustered sources would tend to be protostellar rather than starless. Similarly, if outflows or other protostellar activity trigger 


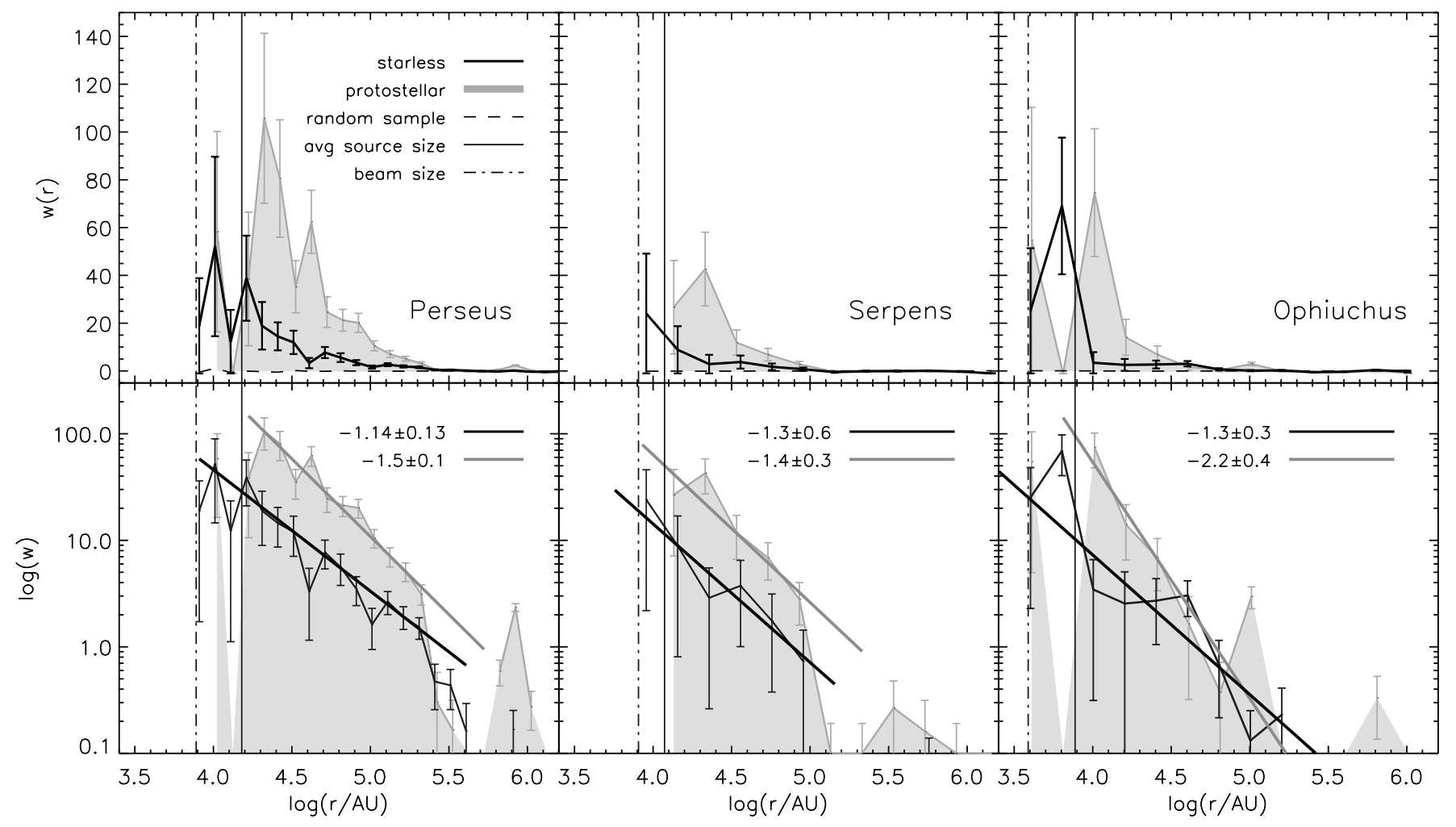

Fig. 11.-Two-point spatial correlation function $w(r)$, plotted as a function of source separation $r$, for starless and protostellar cores in Perseus, Serpens, and Ophiuchus (top panels). Bottom panels plot $\log (w)$, with the best-fitting power-law slope for each distribution. Resolution limits and average source sizes are indicated. Power-law slopes are fit for $r$ larger than the average source size in each cloud. The amplitude of the protostellar $w(r)$ is consistently higher than the starless $w(r)$, indicating that clustering of protostellar cores is stronger on all spatial scales. Slopes for the starless and protostellar populations are similar in each cloud, however, so although the magnitude of clustering differs, the fundamental nature of the clustering does not.

the collapse of nearby cores, we would again expect more protostellar sources in clustered regions. If evolution plays a more important role, on the other hand, the spatial distribution of cores might evolve after protostellar formation, for example as a result of dynamical effects.

\section{ARE THE STARLESS CORES REALLY PRESTELLAR?}

For any discussion of the mass distribution or lifetime of prestellar cores, it is important to determine whether or not our starless cores are likely to be truly prestellar (i.e., will form one or more stars at some point in the future). To this end, we investigate the dynamical state of starless cores by estimating the virial masses of cores in Perseus.

While it is possible that some fraction of the observed starless cores are transient or stable structures that will never form stars, the starless cores detected by our Bolocam surveys have high mean particle densities [typical $\left.n_{1 e 4} \sim(1-3) \times 10^{5} \mathrm{~cm}^{-3} ; \S 3.2\right]$, making them likely to be prestellar (Di Francesco et al. 2007; Keto \& Caselli 2008). Comparison of our data to molecular line observations is a more robust method of determining whether cores are gravitationally bound; line widths provide a direct estimate of the internal energy of cores, while the $1.1 \mathrm{~mm}$ dust masses provide an estimate of the potential energy. An ammonia $\left(\mathrm{NH}_{3}\right)(J, K)=(1,1)$ and $(2,2)$ survey of dense cores in Perseus that includes all of the Bolocam-identified $1.1 \mathrm{~mm}$ cores has recently been completed by Rosolowsky et al. (2008) at the GBT as part of the COMPLETE project. ${ }^{10}$ These observations provide

\footnotetext{
10 "The COMPLETE Survey of Star Forming Regions"; http://cfa-www .harvard.edu/COMPLETE/ (Goodman et al. 2004).
}

a much-needed measure of the temperature and internal motions of dense millimeter cores.

Figure 12 plots the ratio of the dust mass $\left(M_{\text {dust }}\right.$, from Tables 1 and 2$)$ to the virial mass $\left(M_{\text {vir }}\right)$ as a function of $M_{\text {dust }}$ for the 70 cores in Perseus that have well-determined line widths and temperatures from the Rosolowsky et al. (2008) $\mathrm{NH}_{3}$ survey. Although all Bolocam cores were detected in ammonia, not every target had a significant $\mathrm{NH}_{3}(2,2)$ detection or sufficient signalto-noise ratio to measure the line width in the presence of the hyperfine structure. We present the data relevant to Figure 12 in Table 4.

We estimate the virial mass of the cores as

$$
M_{\mathrm{vir}}=\frac{5 \sigma_{\mathrm{H}_{2}}^{2} R}{a G},
$$

where $a$ accounts for the central concentration of the core, $R$ is the radius of the core, and $\sigma_{\mathrm{H}_{2}}$ is the line width of the molecular gas inferred from the ammonia line width. Note that $M_{\text {dust }} / M_{\text {vir }}=$ $1 / \alpha$ for $a=1$, where $\alpha$ is the virial parameter introduced by Bertoldi \& McKee (1992). For simplicity, we adopt a power-law density profile, for which $a=(1-p / 3)(1-2 p / 5)^{-1}$. We report values in Table 4 for a $p=1.5$ profile, giving $a=5 / 4$; for $p=2$ (SIS) the dynamical masses would be $25 \%$ smaller. The total line width of $\mathrm{H}_{2}$ includes thermal and turbulent components, and is calculated as

$$
\sigma_{\mathrm{H}_{2}}^{2}=\sigma_{\mathrm{NH}_{3}}^{2}-\frac{k T_{K}}{17 m_{\mathrm{H}}}+\frac{k T_{K}}{2 m_{\mathrm{H}}} .
$$

The radius is estimated from the $1.1 \mathrm{~mm}$ data as $R=\theta_{\mathrm{dec}} / 2$, or the HWHM. Although the effective radius $R$ appropriate for 


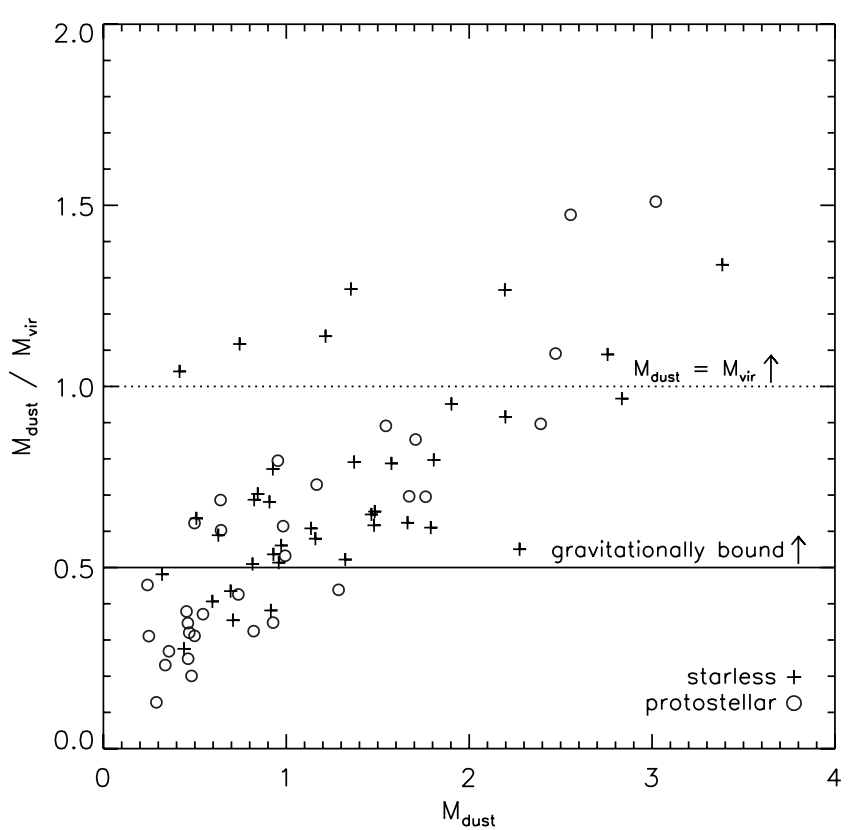

FIG. 12.-Ratio of the total mass derived from dust $\left(M_{\text {dust }}\right)$ to the virial mass $\left(M_{\mathrm{vir}}\right)$, calculated using the temperature and velocity dispersion derived from GBT $\mathrm{NH}_{3}(1,1)$ and $(2,2)$ observations (Rosolowsky et al. 2008), for starless and protostellar cores in Perseus. Only those with well-determined $\mathrm{NH}_{3}$ line widths are shown. Lines indicate the minimum $M_{\text {dust }} / M_{\text {vir }}$ for which cores should be in virial equilibrium (dashed line) or self-gravitating (solid line). [See the electronic edition of the Journal for a color version of this figure.]

the virial theorem is not exactly equivalent to the Gaussian-fit HWHM, we do not expect the correction to be large. If the FWHM is a good representation of $(8 \ln 2)^{1 / 2}$ times the radial dispersion one would measure in the $x-y$ plane for a power-law density profile, then we expect

$$
R=\theta_{\operatorname{dec}} \sqrt{(5-p) /(3-p)} \sqrt{3 / 4} \sqrt{1 /(8 \ln 2)}
$$

or $R \sim 0.56 \theta_{\mathrm{dec}}$ for $p=1.5$, only a $10 \%$ correction.

Dotted and solid lines in Figure 12 indicate regions in the plot for which cores should be virialized and self-gravitating, respectively. Note here that $M_{\text {dust }}=M_{\text {vir }}$ implies $2 K=-U$, where $K$ is the kinetic and $U$ the gravitational energy, while the selfgravitating limit is defined by $K=-U$, or $M_{\text {dust }} / M_{\text {vir }}=0.5$. There are two important points to note here. First, nearly all of the starless cores lie close to or above the self-gravitating line, indicating that they are likely to be gravitationally bound. This statement must be modified by the important caveat that the power-law profile assumed and the method for measuring the dust mass introduce significant systematics that could shift $M_{\text {dust }} / M_{\text {vir }}$ by more than a factor of 2 .

Perhaps more convincingly, there is very little difference between starless and protostellar cores in this plot; even though several of the starless cores lie below the self-gravitating line, this regions is populated by protostellar cores as well, which are by definition capable of forming stars. Furthermore, recent estimates of the dust opacity $\kappa_{1.1 \mathrm{~mm}}$ (Shirley et al. 2007; see $\S 3.2$ ) may increase $M_{\text {dust }}$ by 1.3 , making the starless cores more bound. Based on this discussion, we assume from here on that all starless cores in our $1.1 \mathrm{~mm}$ samples are true prestellar cores.

\section{THE PRESTELLAR CORE MASS DISTRIBUTION AND THE IMF}

One very important measure of the initial conditions of star formation is the prestellar core mass distribution (CMD). In par-
TABLE 4

Dynamical Properties of Perseus Cores

\begin{tabular}{|c|c|c|c|c|c|}
\hline Bolocam ID & $\begin{array}{r}T_{K} \\
(\mathrm{~K})\end{array}$ & $\begin{array}{c}R \\
\left(10^{3} \mathrm{AU}\right)\end{array}$ & $\begin{array}{c}\sigma_{\mathrm{H}_{2}} \\
\left(\mathrm{~km} \mathrm{~s}^{-1}\right)\end{array}$ & $\begin{array}{l}M_{\text {dust }} \\
\left(M_{\odot}\right)\end{array}$ & $\begin{array}{l}M_{\text {vir }} \\
\left(M_{\odot}\right)\end{array}$ \\
\hline Per-Bolo 1 .................... & 9.2 & 3.8 & 0.20 & 0.3 & 0.7 \\
\hline Per-Bolo $2 \ldots \ldots \ldots \ldots \ldots$ & 9.2 & 2.0 & 0.23 & 0.4 & 0.4 \\
\hline Per-Bolo $3 \ldots \ldots \ldots \ldots . . . .$. & 10.0 & 10.7 & 0.22 & 0.9 & 2.4 \\
\hline Per-Bolo 4 .............. & 10.3 & 13.1 & 0.22 & 2.8 & 2.9 \\
\hline Per-Bolo $5 \ldots \ldots \ldots \ldots \ldots$ & 12.1 & 5.9 & 0.26 & 2.6 & 1.7 \\
\hline Per-Bolo $6 \ldots \ldots \ldots \ldots \ldots . . .$. & 9.1 & 13.2 & 0.21 & 3.4 & 2.5 \\
\hline Per-Bolo 11 .................. & 11.2 & 9.1 & 0.27 & 1.8 & 2.9 \\
\hline Per-Bolo $13 \ldots \ldots \ldots . . . .$. & 9.1 & 4.8 & 0.22 & 1.4 & 1.1 \\
\hline Per-Bolo $14 \ldots \ldots \ldots \ldots . . .$. & 9.0 & 4.8 & 0.23 & 1.2 & 1.1 \\
\hline
\end{tabular}

Notes.-Kinetic temperatures $\left(T_{K}\right)$ and line widths $\left(\sigma_{\mathrm{H}_{2}}\right)$ are derived from the $\mathrm{NH}_{3}$ observations of Perseus cores completed by Rosolowsky et al. (2008). The virial mass $M_{\text {vir }}$ is calculated according to eq. (5), with the effective radius $R$ estimated as the deconvolved HWHM of the $1.1 \mathrm{~mm}$ identified core $\left(\theta_{\operatorname{dec}} / 2\right)$. $M_{\text {dust }}$ is the mass calculated from the total $1.1 \mathrm{~mm}$ flux (Tables 1 and 2). Table 4 is published in its entirety in the electronic edition of the Astrophysical Journal. A portion is shown here for guidance regarding its form and content.

ticular, comparing the prestellar CMD to the stellar initial mass function (IMF) provides insight into how the final masses of stars are determined. There are a number of processes that may (jointly) dictate what the final mass of forming star will be. We focus here on two simple cases, assuming that only one drives the shape of the IMF.

If stellar masses are determined by the initial fragmentation into cores, i.e., the final star or binary mass is always a fixed percentage of the original core mass, then the shape of the emergent stellar IMF should closely trace that of the prestellar CMD (e.g., Myers et al. 1998). This might be expected in crowded regions where the mass reservoir is limited to a protostar's nascent core. If, on the other hand, stellar masses are determined by competitive accretion (Bonnell et al. 2001), or by the protostars themselves through feedback mechanisms (e.g., outflows and winds; Shu et al. 1987), we would not expect the emergent IMF to reflect the original core mass distribution (Adams \& Fatuzzo 1996).

\subsection{The Prestellar CMD}

We combine the prestellar core samples from all three clouds, 108 cores in total, and assume $T_{D}=10 \mathrm{~K}$ to calculate masses. As noted above in $\S 4$, our assumption that the majority of starless cores in our sample are truly prestellar is supported by a comparison of the dust mass to the dynamical mass from $\mathrm{NH}_{3} \mathrm{Ob}-$ servations. The resulting prestellar CMD is shown in Figure 13.

The $50 \%$ completeness limit shown $\left(M \sim 0.8 M_{\odot}\right)$ is estimated based on the empirical $50 \%$ completeness curve in Figure 8. Our completeness depends on the size of a given source; as the average core size in Perseus is $68^{\prime \prime}$, we take the $50 \%$ completeness limit for a $70^{\prime \prime}$ FWHM source $\left(M \sim 0.8 M_{\odot}\right)$ to be the average $50 \%$ completeness limit of the sample. The equivalent $90 \%$ completeness limit is a factor of 1.2 higher, $M \sim 0.93 M_{\odot}$. The $50 \%$ completeness limits for average-sized sources are lower in Serpens $\left(0.6 M_{\odot}\right)$ and Ophiuchus $\left(0.5 M_{\odot}\right)$, but as more than half of the total population of prestellar cores are in Perseus, we take $0.8 M_{\odot}$ for the entire prestellar sample.

We fit a power law $\left(d N / d M \propto M^{\alpha}\right)$ to the CMD for $M>$ $0.8 M_{\odot}$, finding a slope of $\alpha=-2.3$, with a reduced $\chi^{2}$ of $\tilde{\chi}^{2}=1.9$. The best-fit slope depends somewhat on the histogram binning, ranging from 2.0 to 2.6 for bin widths of 0.1 to $0.3 M_{\odot}$, so we assign a total uncertainty of 0.4 to our best-fit slope, when 


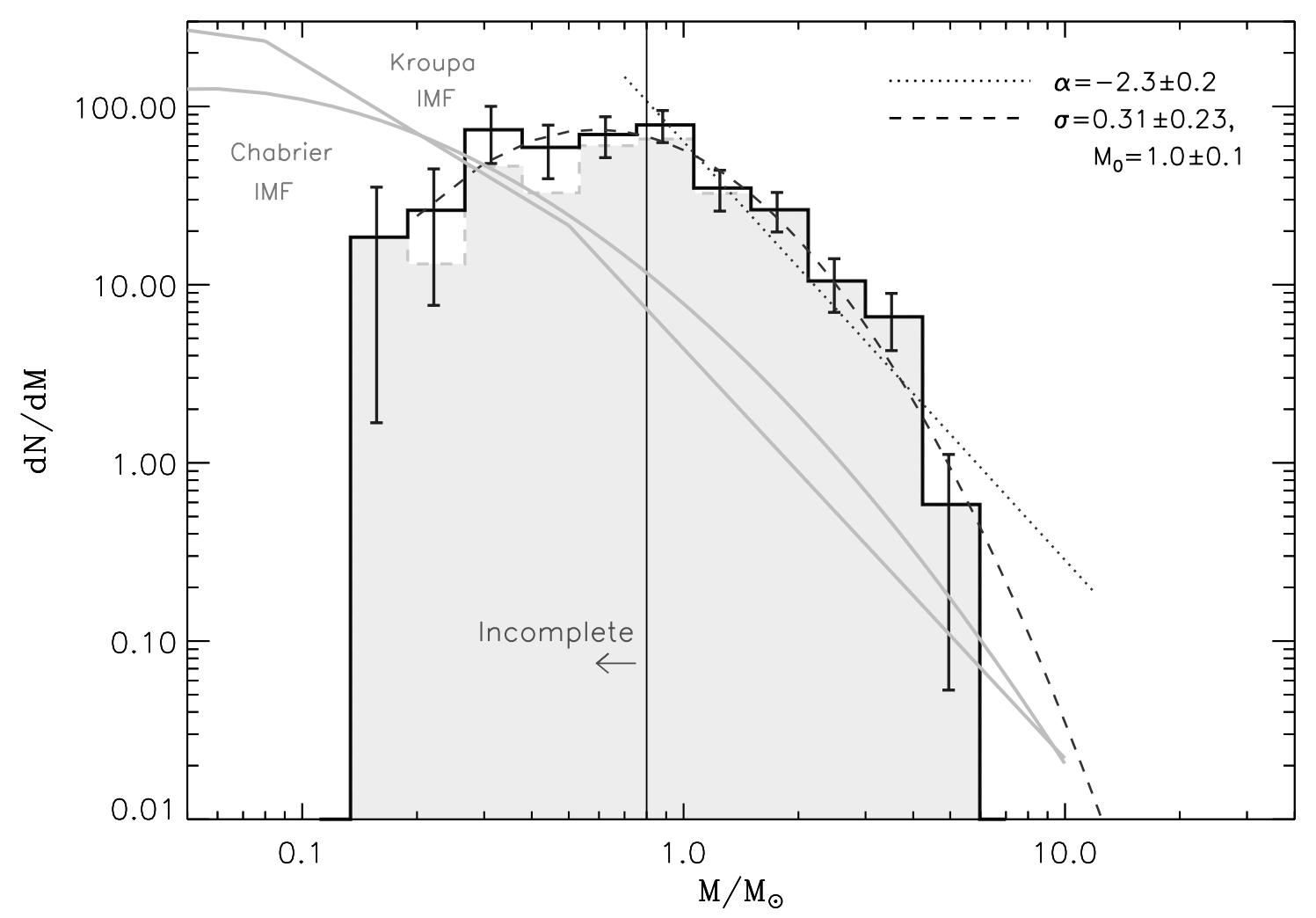

FIG. 13.-Combined prestellar core mass distribution (CMD), with power-law and lognormal fits. The prestellar sample is composed of all starless cores from Perseus, Serpens, and Ophiuchus, and the $50 \%$ mass completeness limit is defined by the completeness limit for average-sized cores in Perseus. Recent measurements of the stellar IMF for $M \gtrsim 0.5 M_{\odot}(\alpha=-2.3$ to -2.8$)$ are similar to the best-fit CMD power-law slope $(\alpha=-2.3 \pm 0.4)$. IMF fits from Chabrier (2005) (lognormal) and Kroupa (2002) (three-component power law) are shown as thick gray lines for reference. The shaded histogram indicates how the mass distribution changes if a small fraction of cores (15\%, based on the six "unbound" cores from Fig. 12) with $M<1 M_{\odot}$ are excluded from the prestellar sample. [See the electronic edition of the Journal for a color version of this figure.]

also taking into account formal fitting errors. We fit a lognormal distribution to $M>0.3 M_{\odot}$, finding a best-fit width $\sigma=0.30 \pm$ 0.03 and characteristic mass $M_{0}=1.0 \pm 0.1 M_{\odot}$. Although the lognormal function is quite a good fit $\left(\tilde{\chi}^{2}=0.5\right)$, the reliability of the turnover in the prestellar CMD is highly questionable given that the completeness limit in Perseus coincides closely with the turnover mass. The prestellar CMD can also be fit by a broken power law with $\alpha=-4.3 \pm 1.1$ for $M>2.5 M_{\odot}$ and $\alpha=-1.7 \pm 0.3$ for $M<2.5 M_{\odot}$, although the uncertainties are large.

Given that source detection is based on peak intensity, we may be incomplete to sources with very large sizes and low surface density even in the higher mass bins $\left(M>0.8 M_{\odot}\right)$. Completeness varies with size similarly to $M \propto R^{2}$; thus the fraction of (possibly) missed sources should decrease with increasing mass. ${ }^{11}$ The effect of missing such low-surface-brightness cores, if they exist and could be considered prestellar, would be to flatten the CMD slightly (i.e., the true slope would be steeper than the observed slope). Instrumental selection effects are discussed further in Paper I.

To be completely consistent, we should exclude the "unbound" cores from Figure 12 (those with $M_{\text {dust }} / M_{\text {vir }}<0.5$ ) from our prestellar CMD. This represents 6 out of the 40 cores that have measured virial masses in Perseus, all 6 of which have $M_{\text {dust }}<$ $1 M_{\odot}$. We do not have virial masses for cores in Serpens or Ophiuchus, but we can randomly remove a similar fraction of sources with $M<1 M_{\odot}$ from each cloud sample (2 sources from

\footnotetext{
11 Unless $M \propto R^{2}$ intrinsically for starless cores, in which case a constant incompleteness fraction would apply over all mass bins.
}

Serpens, 4 from Ophiuchus, and an additional 4 from Perseus). The shaded histogram in Figure 13 indicates how the mass distribution is altered when these 16 "unbound" cores are excluded from the sample. Our derived CMD slope is not affected, as nearly all of the starless cores below the "gravitationally bound" line in Figure 12 have masses below our completeness limit, and even at low masses the CMD is not significantly changed.

There may also be some concern over the use of a single dust temperature $T_{D}=10 \mathrm{~K}$ for all cores. To test the validity of this assumption, we use the kinetic temperatures $\left(T_{K}\right)$ derived from the GBT $\mathrm{NH}_{3}$ survey of Perseus (Rosolowsky et al. 2008; S. Schnee et al. 2008, in preparation) to compute core masses, assuming that the dust and gas are well coupled (i.e., $T_{D}=T_{K}$ ). Figure 14 shows the CMD of prestellar cores in Perseus, both for a single $T_{D}=10 \mathrm{~K}$ and for masses calculated using the $\mathrm{NH}_{3} \mathrm{ki}-$ netic temperatures for each core. There is some change to the shape of the CMD at intermediate masses, but the best-fitting slope for $M>0.8 M_{\odot}(\alpha=-2.3)$ is unchanged. In fact, the deviation from a power law is smaller when using the kinetic temperatures than when using $T_{D}=10 \mathrm{~K}\left(\tilde{\chi}^{2}=1.3\right.$ and 2.5, respectively).

The median $T_{K}$ of prestellar cores in Perseus is $10.8 \mathrm{~K}$, quite close to our adopted $T_{D}=10 \mathrm{~K}$, and the small overall shift in masses (a factor of 1.1) corresponding to an $0.8 \mathrm{~K}$ temperature difference would not affect the derived CMD slope. The dispersion in kinetic temperatures in Perseus is $\pm 2.4 \mathrm{~K}$, or $\pm 0.4 M_{\odot}$ for a $1 M_{\odot}$ core, and the tail of the distribution extends to $T_{K}>$ $15 \mathrm{~K}$ (S. Schnee et al. 2008, in preparation). We do not have temperature information for cores in Serpens or Ophiuchus; if the median temperature were to vary from cloud to cloud by 


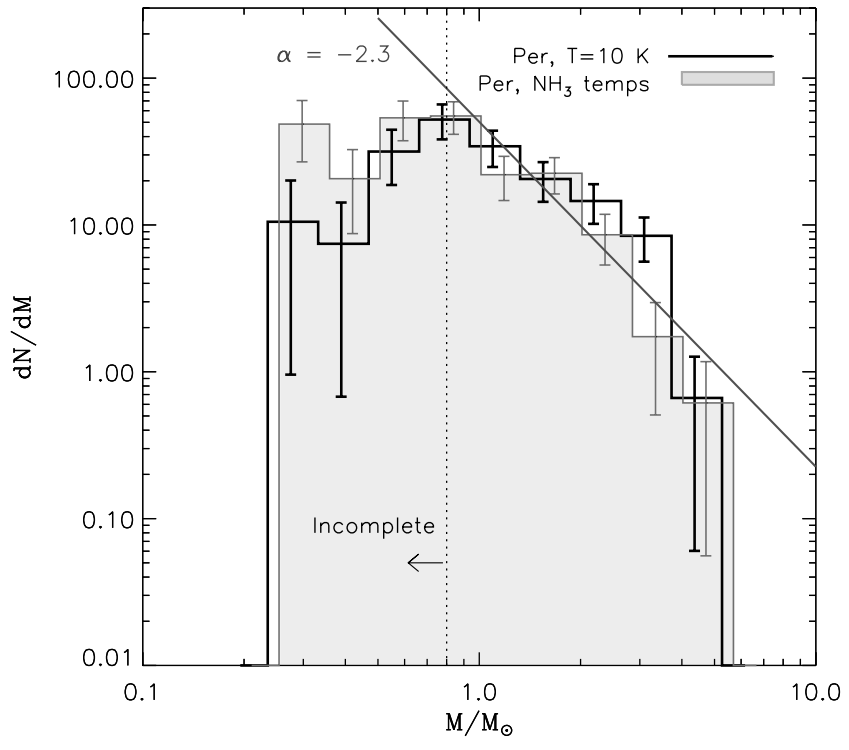

FIG. 14.-Effect on the prestellar CMD in Perseus of using $\mathrm{NH}_{3}$-derived core kinetic temperatures (shaded histogram) rather than assuming a constant $T_{D}=$ $10 \mathrm{~K}$ for all cores (black histogram). Only starless cores in Perseus are included here. The best-fitting power-law slope for $M>0.8 M_{\odot}$ is similar for the two histograms: $\alpha=-2.35 \pm 0.33$ and $\alpha=-2.33 \pm 0.28$ for the $T_{D}=10 \mathrm{~K}$ and $\mathrm{NH}_{3}$ temperature curves, respectively. Although we do not have kinetic temperatures for cores in Serpens or Ophiuchus, we do not expect temperature effects to dramatically alter the derived prestellar CMD slope.

more than a few $\mathrm{K}$, the shape of the combined CMD could be significantly altered. Perhaps a more serious issue is that prestellar cores are not in reality isothermal, but taking into account variations of the temperature with radius requires radiative transfer modeling of each source.

\subsection{Comparison to the $I M F$}

The shape of the local IMF is still uncertain (Scalo 2005), but recent work has found evidence for a slope of $\alpha=-2.3$ to -2.8 for stellar masses $M \gtrsim 1 M_{\odot}$, similar to the slope we measure for the combined prestellar CMD $(\alpha=-2.3 \pm 0.4)$. For example, Reid et al. (2002) find $\alpha=-2.5$ above $0.6 M_{\odot}$, and $\alpha=-2.8$ above $1 M_{\odot}$. Schröder \& Pagel (2003) suggest $\alpha=-2.7$ for $1.1 M_{\odot}<M<1.6 M_{\odot}$ and $\alpha=-3.1$ for $1.6 M_{\odot}<M<4 M_{\odot}$. For reference, the Salpeter IMF has a slope of $\alpha=-2.35$ (Salpeter 1955), and the Scalo (1986) slope for sources with mass $M \gtrsim 1 M_{\odot}$ is $\alpha \sim-2.7$. At lower masses, the IMF flattens, and may be characterized by a lognormal function. Kroupa (2002) suggests a three-component power law for the average singlestar IMF: $\alpha=-2.3$ for $0.5 M_{\odot}<M<1 M_{\odot}, \alpha=-1.3$ for $0.08 M_{\odot}<M<0.5 M_{\odot}$, and $\alpha=-0.3$ for $0.01 M_{\odot}<M<$ $0.08 M_{\odot}$. Chabrier (2005) finds that a lognormal distribution with $\sigma=0.55$ and $M_{0}=0.25 M_{\odot}$ is a good fit for $M<1 M_{\odot}$.

The Kroupa (2002) three-component power law and the Chabrier (2005) lognormal IMFs are shown as thick gray lines in Figure 13. The width of the Chabrier (2005) lognormal $(\sigma=$ $0.55)$ is somewhat larger than the width of the prestellar CMD best-fit lognormal $(\sigma=0.3)$, as expected if we are incomplete at lower masses, and the IMF characteristic mass $\left(M_{0}=0.25 M_{\odot}\right)$ is a factor of 4 lower than that of the $\operatorname{CMD}\left(M_{0}=1.0 M_{\odot}\right)$. A lower characteristic mass for the IMF is expected if some fraction of the core mass is lost in the star formation process. Powerlaw fits for $M>1 M_{\odot}$ appear to be quite similar for the CMD $(\alpha=-2.3 \pm 0.4)$ and IMF $(\alpha=-2.3$ to -2.8$)$, however.
Although we cannot rule out the importance of feedback and other local processes in determining the shape of the IMF, the fact that the prestellar CMD and the local IMF have similar shapes supports a growing body of evidence that the final masses of stars are determined during the core formation process. If this is the case, it is tempting to relate both the prestellar CMD and the IMF to the CMD created from turbulent simulations. Numerical models of turbulent clouds have had some success in reproducing the general shape of the IMF (e.g., Padoan \& Nordlund 2002; Li et al. 2004), but the link is not firmly established. For example, we found in Paper III that the predicted dependence of CMD shape on the turbulent Mach number (BallesterosParedes et al. 2006; Padoan \& Nordlund 2002) does not agree with observations.

Evidence for a direct link between the CMD and the IMF has been found previously based on dust emission surveys of small regions (Testi \& Sargent 1998; Motte et al. 1998), as well as molecular line observations of dense cores (Onishi et al. 2002). Recently, Alves et al. (2007) found evidence for flattening at low masses in the CMD of the Pipe Nebula, as traced by dust extinction toward background stars. Those authors interpret the similarity between the Pipe Nebula CMD and the Trapezium cluster IMF (Muench et al. 2002) as evidence that the stellar IMF is a direct product of the CMD, with a uniform core-to-star efficiency of $30 \% \pm 10 \%$. Although the measured masses of Alves et al. (2007) are somewhat less uncertain than ours because they do not need to assume a dust opacity or temperature, the mean particle densities of cores traced by dust extinction $\left(n \sim 5 \times 10^{3}-2 \times 10^{4} \mathrm{~cm}^{-3}\right)$ are considerably lower than the mean densities of cores traced by our Bolocam $1.1 \mathrm{~mm}$ surveys $\left(n \sim 2 \times 10^{4}-10^{6} \mathrm{~cm}^{-3}\right)$, and they may never form stars. In fact, recent $\mathrm{C}^{18} \mathrm{O}$ and $\mathrm{NH}_{3}$ observations suggest that the dust extinction sources in the Pipe Nebula are pressure-confined, gravitationally unbound starless cores (Lada et al. 2008; Muench et al. 2007). In Orion, Nutter \& Ward-Thompson (2007) find a turnover in the CMD of starless SCUBA $850 \mu \mathrm{m}$ cores at $\sim 1.3 M_{\odot}$. Those authors relate this turnover to a downturn in the Kroupa (2002) IMF at $\sim 0.1 M_{\odot}$, and infer a much lower core-to-star efficiency of $\sim 6 \%$.

If the prestellar CMD does have a one-to-one relationship with the stellar IMF, then the ratio of turnover masses is a measure of the core-collapse efficiency, or the fraction of original core mass that ends up in the final star: $f_{\text {eff }}=M_{\mathrm{TO}}^{\mathrm{IMF}} / M_{\mathrm{TO}}^{\mathrm{CMD}}$. Here $M_{\mathrm{TO}}$ is the mass where the $d N / d M$ distribution, which rises with decreasing mass, flattens out and begins to fall with further decreasing mass. Equivalently, $M_{\mathrm{TO}}^{\mathrm{CMD}} / M_{\mathrm{TO}}^{\mathrm{IMF}}=1-f_{\mathrm{eff}}$ is the fraction of core mass lost in the star formation process.

Our limited completeness dictates that we can only measure a lower limit to $f_{\text {eff }}$. If there is a true turnover in the prestellar $\mathrm{CMD}$, it must occur below our completeness limit, at $M_{\mathrm{TO}}^{\mathrm{CMD}} \lesssim$ $1.0 M_{\odot}$. The system IMF, i.e., treating binaries and multiple systems as single rather than multiple objects, peaks at $M_{\mathrm{TO}}^{\mathrm{IMF}} \sim$ 0.2-0.3 $M_{\odot}$ (e.g., Chabrier 2005; Luhman et al. 2003). This system IMF is appropriate for comparison to our CMDs, as we would not resolve such multiple systems even if they form from distinct cores. For an IMF turnover mass of $0.25 M_{\odot}, M_{\mathrm{TO}}^{\mathrm{CMD}} \lesssim$ $1.0 M_{\odot}$ implies that at least $25 \%$ of the initial core mass is accreted onto the final star or stellar system. Characteristic masses associated with lognormal fits to both the IMF and CMD imply a similar ratio of $f_{\text {eff }} \gtrsim M_{0}^{\mathrm{IMF}} / M_{0}^{\mathrm{CMD}} \sim 0.25 / 1.0 \sim 0.25$.

Our conclusion that the core-to-star efficiency is at least $25 \%$ is consistent with the value found by Alves et al. (2007) for the Pipe Nebula (30\%), and with predicted efficiencies of $25 \%-75 \%$ 
from recent analytic models of bipolar protostellar outflows (Matzner \& McKee 2000).

\subsection{The Effect of "Multiple" Sources}

As noted in $\S 3,17 \%-55 \%$ of our protostellar cores are associated with more than one (two to three) embedded protostars. We can expect that a similar fraction of prestellar cores will form a resolved binary or multiple system, making a direct mapping between the CMD and IMF difficult to justify unless the resolutions are matched. Given the relatively low resolution of the Bolocam data (30"), some cores must result in wide-separation multiple stellar systems that would not be considered single objects in the system IMF. Furthermore, the system IMF likely evolves over time due to dynamical effects such as the decay of high-order multiples, ejections, and close interactions (see Goodwin et al. 2008 for more discussion of the CMD to IMF mapping).

The effect of these multiple sources will likely be to steepen the CMD, as higher mass cores are divided into multiple lower mass sources. This is consistent with the fact that the prestellar CMD slope is at the low end of the IMF slope range. It is important to keep in mind that we would expect only $\sim 25 \%-30 \%$ of cores in the combined prestellar CMD to be "multiple" sources, so they should not dominate the CMD slope. While we know that most stars occur in binaries (Duchêne et al. 2007 and references therein), and thus that there must be some unresolved binaries in the Spitzer data, these compact systems will most likely be unresolved in the system IMF as well, so they do not affect our comparison.

\subsection{The Protostellar Core Mass Distribution}

For comparison, we show in Figure 15 the mass distribution of the combined protostellar core sample from all three clouds, assuming $T_{D}=15 \mathrm{~K}$. The protostellar CMD is considerably wider and flatter than the prestellar CMD, and extends to higher masses. The best-fitting power-law slope $\left(\alpha=-1.8 ; \tilde{\chi}^{2}=1.7\right)$ is shallower than for the prestellar sample, and the best-fitting lognormal distribution ( $\sigma=0.51 \pm 0.07, M_{0}=0.8 \pm 0.2 ; \tilde{\chi}^{2}=1.3$ ) is wider by nearly a factor of 2 . The protostellar CMD must be interpreted in the context of a constant assumed $T_{D}=15 \mathrm{~K}$; an intrinsic spread of temperatures would tend to flatten the mass distribution even more, but $15 \mathrm{~K}$ has been determined to be the best single $T_{D}$ based on many detailed radiative transfer models (Shirley et al. 2002; Young et al. 2003).

A protostellar CMD that extends to lower masses than the prestellar CMD is not unexpected, given that some fraction of the core mass has already been accreted onto the central source for protostellar cores. An extension to higher masses in the protostellar $\mathrm{CMD}$ as compared to the prestellar CMD, however, can only be explained if (1) we are underestimating the dust temperature of protostellar sources or underestimating the total mass of prestellar cores, (2) the current populations of prestellar cores in these clouds have lower mass than the generation of cores that have already formed protostars, or (3) the highest mass prestellar cores collapse to form protostars very quickly.

Hatchell \& Fuller (2008) examine the relationship between starless and protostellar mass distributions in Perseus based on SCUBA data, finding that a simple mass-dependent evolutionary model in which higher mass cores form protostars on a shorter timescale than lower mass cores can qualitatively explain the observed steeper prestellar CMD slope, and extension of the protostellar CMD to higher masses. Another step toward resolving the reasons for these differences may be careful radiative

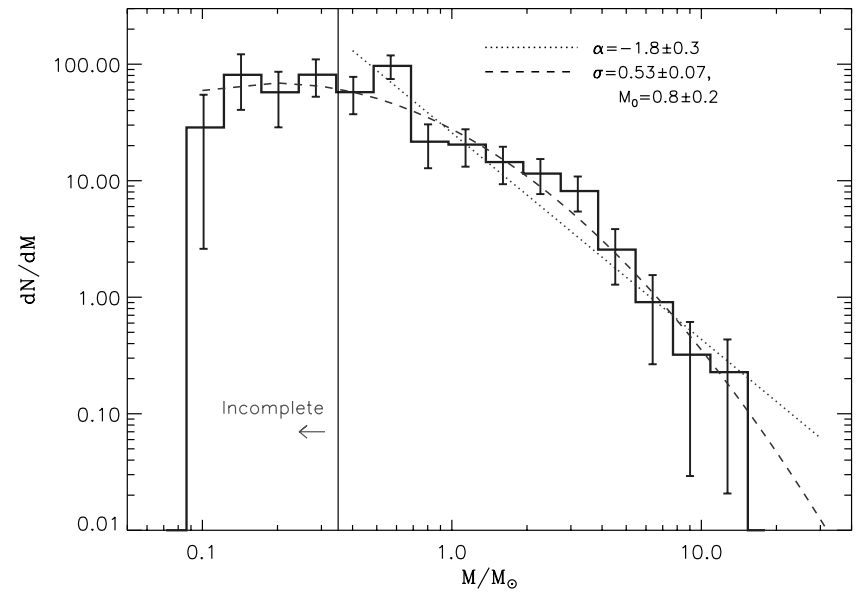

FIG. 15.-Combined protostellar mass distribution, with power-law and lognormal fits. The protostellar CMD includes all protostellar cores from Perseus, Serpens, and Ophiuchus, and the 50\% mass completeness limit is defined by the completeness to average-sized protostellar cores in Perseus. The best-fit power law $(\alpha=-1.8)$ is shallower, and the distribution as a whole is wider, than the prestellar CMD (Fig. 13). This difference is expected if the protostellar CMD evolved from the prestellar CMD. The fact that the protostellar distribution extends to higher masses than the prestellar CMD is more difficult to explain, however, and may suggest that higher mass prestellar cores are relatively short lived. [See the electronic edition of the Journal for a color version of this figure.]

transfer models of each prestellar and protostellar source to replace the assumption of a constant $T_{D}$.

\section{LIFETIME OF THE PRESTELLAR PHASE}

We can use the relative number of prestellar cores and embedded protostars to estimate the lifetime of the prestellar core phase, which is essential for understanding the physical processes that govern the formation, support, and collapse of star-forming cores. Several assumptions are required for this calculation. We assume that all of the starless cores in our sample are in fact prestellar, and will eventually collapse to form stars or brown dwarfs (see $\S 4$ ). As we ultimately calibrate our lifetimes based on the Class II phase $\left(2 \times 10^{6}\right.$ yr; Kenyon et al. 1990; Cieza et al. 2007; Spezzi et al. 2008), star formation must have been steady in time for at least the last $2 \mathrm{Myr}$. In addition, we must assume that there is no significant evolutionary dependence on source mass. Although this kind of analysis relies on a number of assumptions, it has the advantage of being quite simple, and it does not require on an a priori accretion rate or star formation model.

Table 5 lists the ratio of the number of starless cores $\left(N_{\mathrm{SL}}\right)$ to embedded protostars $\left(N_{\text {emb }}=N_{\text {Class } 0}+N_{\text {Class I }}\right)$ for all three clouds, and the starless core lifetime $\left(t_{\mathrm{SL}}\right)$ derived from that ratio: $t_{\mathrm{SL}}=$ $t_{\mathrm{emb}}\left(N_{\mathrm{SL}} / N_{\mathrm{emb}}\right)$. The number of embedded protostars in each cloud is derived in a companion paper (M. L. Enoch et al. 2008, in preparation), in which the SED derived from c2d Spitzer photometry and $1.1 \mathrm{~mm}$ fluxes is used to classify protostars based on their bolometric temperature, $T_{\text {bol }}$ : Class $0\left(T_{\text {bol }}<70 \mathrm{~K}\right)$ and Class I ( $70 \mathrm{~K}<T_{\text {bol }}<650 \mathrm{~K}$ ). In addition to the $T_{\text {bol }}$ classification, all embedded protostars are required to be detected at $1.1 \mathrm{~mm}$, so that we are complete to embedded sources with $M_{\text {env }} \gtrsim 0.1 M_{\odot}$ (M. L. Enoch et al. 2008, in preparation).

The ratio of the number of starless cores to the number of embedded protostars is $N_{\mathrm{SL}} / N_{\mathrm{emb}}=1.0$ in Perseus, 0.4 in Serpens, and 0.8 in Ophiuchus. For an embedded protostar phase that lasts $t_{\mathrm{emb}}=5.4 \times 10^{5} \mathrm{yr}$ (N. J. Evans et al. 2008 , in preparation), the observed ratios imply prestellar core lifetimes of $5 \times 10^{5} \mathrm{yr}$ in Perseus, $2 \times 10^{5} \mathrm{yr}$ in Serpens, and $5 \times 10^{5} \mathrm{yr}$ in Ophiuchus. 
TABLE 5

Lifetime of the Prestellar Core Phase

\begin{tabular}{|c|c|c|c|c|c|}
\hline Cloud & $N_{\mathrm{SL}} / N_{\mathrm{emb}}$ & $\begin{array}{l}t_{\mathrm{SL}} \\
(\mathrm{yr})\end{array}$ & $\begin{array}{l}\left\langle n_{1 e 4}\right\rangle_{\mathrm{SL}} \\
\left(\mathrm{cm}^{-3}\right)\end{array}$ & $\begin{array}{c}t_{\mathrm{ff}} \\
(\mathrm{yr})\end{array}$ & $t_{\mathrm{SL}} / t_{\mathrm{ff}}$ \\
\hline 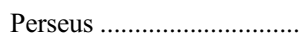 & $67 / 66=1.0$ & $5 \times 10^{5}$ & $1.4 \times 10^{5}$ & $1.4 \times 10^{5}$ & 3.9 \\
\hline 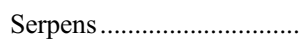 & $15 / 35=0.4$ & $2 \times 10^{5}$ & $1.2 \times 10^{5}$ & $1.5 \times 10^{5}$ & 1.5 \\
\hline 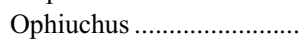 & $26 / 28=0.9$ & $5 \times 10^{5}$ & $1.6 \times 10^{5}$ & $1.3 \times 10^{5}$ & 3.9 \\
\hline 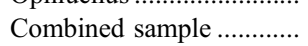 & $108 / 129=0.8$ & $4.5 \times 10^{5}$ & $1.7 \times 10^{5}$ & $1.4 \times 10^{5}$ & 3.2 \\
\hline Low density ...................... & $78 / 129=0.6$ & $3.3 \times 10^{5}$ & $1.3 \times 10^{5}$ & $1.5 \times 10^{5}$ & 2.2 \\
\hline High density................... & $30 / 129=0.2$ & $1.3 \times 10^{5}$ & $2.8 \times 10^{5}$ & $1.0 \times 10^{5}$ & 1.3 \\
\hline
\end{tabular}

Notes. $-N_{\text {emb }}$ refers to the total number of embedded protostars, i.e., those in the Class 0 and Class I phases: $N_{\text {emb }}=N_{\text {Class } 0}+N_{\text {Class I }}$. The number of embedded protostars is derived in a companion paper (M. L. Enoch et al. 2008, in preparation). The prestellar core lifetime $t_{\mathrm{SL}}$ is derived from the number of starless cores: $t_{\mathrm{SL}}=$ $t_{\mathrm{emb}}\left(N_{\mathrm{SL}} / N_{\mathrm{emb}}\right)$, for an embedded phase lifetime $t_{\mathrm{emb}}=5.4 \times 10^{5} \mathrm{yr}$ (N. J. Evans et al. 2008, in preparation). Core mean densities $n_{1 e 4}$ are calculated in a fixed linear aperture of diameter $10^{4} \mathrm{AU}$, and the free-fall timescale $t_{\mathrm{ff}}$ of starless cores is derived from the typical mean density $\left\langle n_{1 e 4}\right\rangle_{\mathrm{SL}}$ of the starless samples using eq. (8). The "combined sample" includes sources from all three clouds; this combined sample is divided into "low density" and "high density" bins representing cores with $n_{1 e 4}<2 \times 10^{5}$ and $>2 \times 10^{5} \mathrm{~cm}^{-3}$, respectively.

Note that we also found approximately equal numbers of protostellar and starless cores in all three clouds (Table 3), further confirmation that the lifetime of starless cores is similar to that of the embedded protostellar phase. ${ }^{12}$ Therefore, the dense starless cores we are sensitive to last for $(2-5) \times 10^{5} \mathrm{yr}$ in all three clouds. The differences in $N_{\mathrm{SL}} / N_{\text {emb }}$ from cloud to cloud may be environmental. The ratio is lowest in Serpens, which has the highest mass cores on average. As noted in $\S 5.4$, higher mass cores tend to be protostellar, possibly because they collapse on a shorter timescale than lower mass cores.

Taking all three clouds together yields a prestellar core lifetime of $4.5 \times 10^{5} \mathrm{yr}$. The uncertainty in the measured number of prestellar cores is approximately \pm 10 , based on the range in the number of starless cores for different identification criteria (see $\S 2.3)$. Given a similar uncertainty in the number of embedded protostars (see M. L. Enoch et al. 2008, in preparation), this corresponds to an uncertainty in the lifetime of prestellar cores of approximately $0.8 \times 10^{5} \mathrm{yr}$.

Published measurements of the prestellar core lifetime vary by 2 orders of magnitude, from a few $\times 10^{5}$ to $10^{7} \mathrm{yr}$ (WardThompson et al. 2007). For example, Lee \& Myers (1999) calculate a core lifetime of $6 \times 10^{5} \mathrm{yr}$ for optically selected cores with mean densities of $(6-8) \times 10^{3} \mathrm{~cm}^{-3}$, while Jessop \& WardThompson (2000) find a lifetime of $10^{7}$ yr for low-density cores detected from column density maps based on IRAS far-infrared observations. Our results are similar to recent findings in Perseus by Jørgensen et al. (2007) and Hatchell et al. (2007), both of whom find approximately equal lifetimes for the starless and embedded protostellar phases by comparing SCUBA $850 \mu \mathrm{m}$ maps with Spitzer $\mathrm{c} 2 \mathrm{~d}$ data. The approximate equality between $N_{\mathrm{SL}}$ and $N_{\mathrm{PS}}$ has been found by a number of studies, and was noted early on by Beichman et al. (1986) for the $\mathrm{NH}_{3}$ cores of Myers \& Benson (1983). Our results are also consistent within a factor of 2 with the lifetime derived by Visser et al. (2002) for a sample of Lynds dark clouds observed with SCUBA.

The average mean density (calculated in a fixed linear aperture of $10^{4} \mathrm{AU}$; see $\S 3.2$ ) of the starless core samples, $\left\langle n_{1 e 4}\right\rangle_{\mathrm{SL}}$, and

\footnotetext{
12 The $N_{\mathrm{SL}} / N_{\text {emb }}$ ratios in Table 5 differ from $N_{\mathrm{SL}} / N_{\mathrm{PS}}$ in Table 3 due to the presence of multiple embedded protostars in some protostellar cores $(\S 3)$ and to the fact that some embedded protostars are "band filled" at $1.1 \mathrm{~mm}$. Band-filled sources are not associated with a distinct core, but appear to be associated with millimeter emission that is either extended or below the $5 \sigma$ detection limit.
}

corresponding free-fall timescale, $t_{\mathrm{ff}}$, are also given in Table 5 . The free-fall time is the timescale on which starless cores will collapse in the absence of internal support, and is calculated from the mean particle density (Spitzer 1978):

$$
t_{\mathrm{ff}}=\sqrt{\frac{3 \pi}{32 G \rho}}=\sqrt{\frac{3 \pi}{32 G\langle n\rangle \mu_{p} m_{\mathrm{H}}}},
$$

where $m_{\mathrm{H}}$ is the mass of hydrogen and $\mu_{p}=2.33$ is the mean molecular weight per particle. Mean densities are similar in all three clouds, $\left\langle n_{1 e 4}\right\rangle_{\mathrm{SL}}=(1-2) \times 10^{5} \mathrm{~cm}^{-3}$, with corresponding free-fall times of $t_{\mathrm{ff}}=(1.3-1.5) \times 10^{5} \mathrm{yr}$. The final column in Table 5 gives the ratio of the measured starless core lifetime to the average free-fall timescale in each cloud: $t_{\mathrm{SL}} / t_{\mathrm{ff}}=1.5-3.9$. Thus prestellar cores last for only a few free-fall times in all three clouds.

Such a short prestellar core lifetime argues for a dynamic, rather than quasi-static, core evolutionary scenario. One classical quasi-static model is that of magnetically dominated star formation, in which the evolution of highly subcritical cores is moderated by ambipolar diffusion (Shu et al. 1987). In this paradigm, prestellar cores should have lifetimes similar to the ambipolar diffusion timescale, or $t_{\mathrm{AD}} \sim 7 \times 10^{6} \mathrm{yr}$ for typical ionization levels in low-mass star forming regions (e.g., Nakano 1998; Evans 1999), more than an order of magnitude longer than our results.

It is important to emphasize here that our Bolocam surveys are sensitive to cores with relatively high mean density $[n \gtrsim(2-3) \times$ $10^{4} \mathrm{~cm}^{-3}$; Paper III]. ${ }^{13}$ Thus we may be sampling only the densest end stage in a longer core evolutionary picture, in which case a magnetic-field-dominated scenario could still be applicable at early times (e.g., Tassis \& Mouschovias 2004). Ciolek \& Basu (2001) also note that $t_{\mathrm{AD}}$ can be as short as a few free-fall times for marginally subcritical cores, and Galván-Madrid et al. (2007) find approximately equal numbers af starless and protostellar cores, consistent with our observations, in simulations of magnetically supercritical, turbulent, isothermal molecular clouds.

\footnotetext{
${ }^{13}$ Note that this limiting density takes into account the $50 \%$ completeness limit to starless cores as discussed in $\S \S 3.3$ and 5.1 ; it is the typical density found when one calculates a mean density along the $50 \%$ completeness curve as a function of size in Figs. 8 and 9.
} 


\subsection{Density Dependence}

Interestingly, if we divide our sample into two density bins, $n_{1 e 4}<2 \times 10^{5}$ and $>2 \times 10^{5} \mathrm{~cm}^{-3}$, we find a longer lifetime for lower density cores: $3.3 \times 10^{5}$ versus $1.3 \times 10^{5} \mathrm{yr}$ for the higher density sample (see Table 5 ). This result suggests that the prestellar core lifetime becomes shorter as cores become more centrally condensed, at a faster rate than $n^{-0.5}$ (i.e., that expected based on the dependence of $t_{\mathrm{ff}}$ on $n$; see eq. [8]). The observed trend, $t_{\mathrm{SL}} \propto n^{-1.2}$, is closer to the $n^{-0.85}$ dependence suggested by Jessop \& Ward-Thompson (2000).

\subsection{Additional Uncertainties}

The prestellar lifetime depends on the ratio with protostellar cores, but our sensitivities to prestellar and protostellar sources are not equal due to the different assumed dust temperatures: the protostellar core mass completeness limit is lower by approximately a factor of 2 . Of the 55 protostellar cores in Perseus, 13 fall between the protostellar and starless completeness limits (similarly 3/20 in Serpens, and 5/17 in Ophiuchus), suggesting that the prestellar core lifetime should perhaps be increased by a factor of 1.3 .

We must also consider the possible bias introduced by using the number of embedded protostars, as detected with the higher resolution Spitzer data, rather than the number of Bolocam-detected protostellar cores, to calculate the prestellar lifetime. $N_{\mathrm{emb}} / N_{\mathrm{PS}}=$ 1.2, 1.8, and 1.6 in Per, Ser, and Oph, respectively, as can be seen by comparing Tables 3 and 5. If the starless cores have a similar "multiplicity" fraction that we miss due to the 30 " resolution of Bolocam, then the prestellar lifetimes would be increased by these factors. If cores fragment at later times, or if multiple protostars collapse from a single core, our lifetimes would not be affected.

On the other hand, if some fraction of the starless core sample are not prestellar but unbound starless cores, our calculated lifetime of $4.5 \times 10^{5} \mathrm{yr}$ would be an overestimate of the true prestellar core lifetime. Extrapolating from the six cores with $M_{\text {dust }} / M_{\text {vir }}<$ 0.5 in Figure 12, the lifetime could decrease by a factor of 1.2. Likewise, the assumed embedded phase lifetime is based on the ratio of Class 0 and Class I protostars to Class II sources; if a large fraction of the Class I sources from N. J. Evans et al. (2008, in preparation) are not embedded protostars but edge-on $\mathrm{T}$ Tauri disks (e.g., Crapsi et al. 2008), the embedded phase lifetime could be as low as $2.8 \times 10^{5} \mathrm{yr}$, decreasing the prestellar core lifetime by a factor of 2 .

Finally, the assumption of a simple continuous flow of star formation for the last 2 Myr may, of course, be incorrect, but without a detailed star formation rate model (which might be equally incorrect) it is the best we can do. We hope to mitigate errors from variations in the star formation rate by averaging over three clouds. Given the range of possible errors, we do not apply correction factors to the numbers in Table 5, but consider the absolute uncertainty in the prestellar core lifetime to be a factor of 2 in either direction.

\section{CONCLUSIONS}

Utilizing large-scale $1.1 \mathrm{~mm}$ surveys (Enoch et al. 2006; Young et al. 2006; Enoch et al. 2007) together with Spitzer IRAC and MIPS maps from the c2d Legacy program (Evans et al. 2003), we have carried out an unbiased census of prestellar and protostellar cores in the Perseus, Serpens, and Ophiuchus molecular clouds. We identify a total of 108 starless and 92 protostellar cores in the three cloud sample. Based on a comparison of $1.1 \mathrm{~mm}$ derived masses to virial masses derived from an $\mathrm{NH}_{3}$ survey of Perseus cores (Rosolowsky et al. 2008), we conclude that the majority of our starless cores are likely to be gravitationally bound, and thus prestellar.

The spatial distributions of both starless and protostellar cores are similar in these three molecular with varying global properties. In all three clouds both starless and protostellar cores are found only at relatively high cloud column densities: $75 \%$ of cores are associated with $A_{V} \gtrsim 6.5-9.5 \mathrm{mag}$ in Perseus, $A_{V} \gtrsim 6-10 \mathrm{mag}$ in Serpens, and $A_{V} \gtrsim 19.5-25.5 \mathrm{mag}$ in Ophiuchus. Spatial clustering of starless cores is similar in nature to protostellar cores but lower in amplitude, based on the two-point spatial correlation function and peak surface density of cores.

Cloud environment does appear to have some effect on the physical properties of starless cores, however, and how they differ from cores that have already formed protostars. Starless cores in Perseus are larger and have lower mean densities than protostellar cores; we suggest a simple scenario by which protostellar cores might have evolved from starless cores in that cloud, becoming smaller and denser at a fixed mass. In Serpens, it appears that future star formation will occur in lower mass cores than those that are currently forming protostars. Meanwhile, in Ophiuchus we see essentially no difference between cores that have formed stars and those that have not. Of the three clouds, Serpens has the highest mean cloud density (measured within the $A_{V}=2$ contour; Enoch et al. 2007) and the highest turbulent Mach number (Enoch et al. 2007; J. Pineda 2006, private communication), which may be related to its low fraction of starless cores $\left(N_{\mathrm{SL}} / N_{\mathrm{emb}}=0.4\right.$ compared to 1.0 in the other clouds $)$ and the fact that the higher mass cores have already formed protostars

The combined prestellar CMD, which includes 108 prestellar cores from three clouds, has a slope above our completeness limit $\left(0.8 M_{\odot}\right)$ of $\alpha=-2.3 \pm 0.4$. This result is consistent with recent measurements of the stellar initial mass function $(\alpha=-2.3$ to -2.8; e.g., Reid et al. 2002; Kroupa 2002), providing further evidence that the final masses of stars are directly linked to the core formation process. We place a lower limit on the core collapse efficiency (the percentage of initial core mass that ends up in the final star) of $25 \%$. A more secure link between the CMD and IMF requires measurement of the CMD down to masses less than $0.2 M_{\odot}$ in samples of cores that can be demonstrated to be likely prestellar.

In all three clouds the lifetime of dense prestellar cores is similar to the lifetime of embedded protostars, or $(2-5) \times 10^{5} \mathrm{yr}$. The three-cloud average is $(4.5 \pm 0.8) \times 10^{5} \mathrm{yr}$ (with an absolute uncertainty of a factor of 2), arguing strongly for dynamic core evolution on a few free-fall timescales. Such a short prestellar core lifetime is inconsistent with highly magnetically subcritical cores, in which case evolution should occur over an ambipolar diffusion timescale $\left(t_{\mathrm{AD}} \sim 10^{7} \mathrm{yr}\right.$; e.g., Nakano 1998). Our results suggest, rather, a dynamic core evolutionary scenario, as might be appropriate if turbulence dominates the cloud physics (Mac Low $\&$ Klessen 2004), or for near-critical magnetic models (Ciolek \& Basu 2001). The observed prestellar core lifetime decreases with increasing mean density, at a rate faster than that expected from the dependence of the free-fall timescale on density.

Although this measurement of the prestellar core lifetime supports a dynamic paradigm over a quasi-static one, the distinction is not clear-cut. Our observations could still be consistent with a quasi-static picture if we are only observing the densest stages $\left(n>2 \times 10^{4} \mathrm{~cm}^{-3}\right)$ of a longer scale core evolution. Furthermore, the fact that we observe extinction thresholds for finding dense cores at $A_{V} \gtrsim 6$ mag may be a hint that magnetic fields become important in the low-column-density regions of molecular clouds, inhibiting the formation of high-density prestellar cores (and thus star formation). Better measurements of magnetic field strengths 
and more detailed comparisons between observations and models will be help to resolve these ambiguities.

The authors are grateful to Jens Kauffman and Jason Kirk for their insightful comments and suggestions, and to the anonymous referee for raising questions and issues that helped to improve this work. Support for this work, part of the Spitzer Legacy Science Program, was provided by NASA through contracts 1224608 and
1230782 issued by the Jet Propulsion Laboratory, California Institute of Technology, under NASA contract 1407. Additional support was provided by NASA through the Spitzer Space Telescope Fellowship Program and obtained from NASA Origins grant NNG04GG24G to the University of Texas at Austin. Support for the development of Bolocam was provided by NSF grants AST 99-80846 and AST 02-06158. M. L. E. acknowledges support of a Caltech Moore Fellowship and a Spitzer Space Telescope Postdoctoral Fellowship.
Adams, F. C., \& Fatuzzo, M. 1996, ApJ, 464, 256

Alves, J., Lada, C. J., \& Lada, E. A. 2001, Nature, 409, 159

Alves, J., Lombardi, M., \& Lada, C. J. 2007, A\&A, 462, L17

André, P., Ward-Thompson, D., \& Barsony, M. 1993, ApJ, 406, 122

Ballesteros-Paredes, J., Gazol, A., Kim, J., Klessen, R. S., Jappsen, A.-K., \& Tejero, E. 2006, ApJ, 637, 384

Ballesteros-Paredes, J., Klessen, R. S., \& Vázquez-Semadeni, E. 2003, ApJ, 592, 188

Beichman, C. A., Myers, P. C., Emerson, J. P., Harris, S., Mathieu, R., Benson, P. J., \& Jennings, R. E. 1986, ApJ, 307, 337

Bertoldi, F., \& McKee, C. F. 1992, ApJ, 395, 140

Bonnell, I. A., Bate, M. R., Clarke, C. J., \& Pringle, J. E. 2001, MNRAS, 323, 785

Bonnor, W. B. 1956, MNRAS, 116, 351

Chabrier, G. 2005, in The Stellar Initial Mass Function Fifty Years Later, ed. E. Corbelli, F. Palla, \& H. Zinnecker (Dordrecht: Springer), 41

Cieza, L., et al. 2007, ApJ, 667, 308

Ciolek, G. E., \& Basu, S. 2001, ApJ, 547, 272

Crapsi, A., van Dishoeck, E. F., Hogerheijde, M. R., Pontoppidan, K. M., \& Dullemond, C. P. 2008, A\&A, 486, 245

Crutcher, R. M. 1999, ApJ, 520, 706

Di Francesco, J., Evans, N. J., II, Caselli, P., Myers, P. C., Shirley, Y., Aikawa, Y., \& Tafalla, M. 2007, in Protostars and Planets V, ed. B. Reipurth, D. Jewitt, \& K. Keil (Tucson: Univ. Arizona Press), 17

Duchêne, G., Delgado Donate, E., Haisch, K. E., Jr., Loinard, L., \& Rodriguez, L. F. 2007, in Protostars and Planets V, ed. B. Reipurth, D. Jewitt, \& K. Keil (Tucson: Univ. Arizona Press), 379

Dunham, M. M., Crapsi, A., Evans, N. J., II, Bourke, T. L., Huard, T. L., Myers, P. C., \& Kauffmann, J. 2008, ApJS, in press (arXiv:0806.1754)

Ebert, R. 1955, Z. Astrophys., 37, 217

Enoch, M. L., Glenn, J., Evans, N. J., II, Sargent, A. I., Young, K. E., \& Huard, T. L. 2007, ApJ, 666, 982 (Paper III)

Enoch, M. L., et al. 2006, ApJ, 638, 293 (Paper I)

Evans, N. J., II. 1999, ARA\&A, 37, 311

Evans, N. J., II, Rawlings, J. M. C., Shirley, Y. L., \& Mundy, L. G. 2001, ApJ, 557,193

Evans, N. J., II, et al. 2003, PASP, 115, 965

2007, Final Delivery of Data from the c2d Legacy Project: IRAC and MIPS (Austin: Univ. Texas Dept. Astron.)

Frerking, M. A., Langer, W. D., \& Wilson, R. W. 1982, ApJ, 262, 590

Galván-Madrid, R., Vázquez-Semadeni, E., Kim, J., \& Ballesteros-Paredes, J. 2007, ApJ, 670, 480

Goodman, A. A., \& the COMPLETE Team. 2004, in Star Formation in the Interstellar Medium, ed. D. Johnstone et al. (San Francisco: ASP), 171

Goodwin, S. P., Nutter, D., Kroupa, P., Ward-Thompson, D., \& Whitworth, A. P. 2008, A\&A, 477, 823

Gregersen, E. M., \& Evans, N. J., II. 2000, ApJ, 538, 260

Harvey, P. M., Merin, B., Huard, T. L., Rebull, L. M., Chapman, N., Evans, N. J., II, \& Myers, P. C. 2007a, ApJ, 663, 1149

Harvey, P. M., et al. 2006, ApJ, 644, 307 $2007 \mathrm{~b}$, ApJ, 663, 1139

Hatchell, J., \& Fuller, G. 2008, A\&A, 482, 855

Hatchell, J., Fuller, G. A., Richer, J. S., Harries, T. J., \& Ladd, E. F. 2007, A\&A, 468, 1009

Huard, T. L., et al. 2006, ApJ, 640, 391

Jessop, N. E., \& Ward-Thompson, D. 2000, MNRAS, 311, 63

Johnstone, D., Wilson, C. D., Moriarty-Schieven, G., Joncas, G., Smith, G., Gregersen, E., \& Fich, M. 2000, ApJ, 545, 327

Jørgensen, J. K., Johnstone, D., Kirk, H., \& Myers, P. C. 2007, ApJ, 656, 293

Jørgensen, J. K., et al. 2006, ApJ, 645, 1246

\section{EFERENCES}

Kenyon, S. J., Hartmann, L. W., Strom, K. M., \& Strom, S. E. 1990, AJ, 99, 869

Keto, E., \& Caselli, P. 2008, ApJ, 683, 238

Kroupa, P. 2002, Science, 295, 82

Lada, C. J., \& Lada, E. A. 2003, ARA\&A, 41, 57

Lada, C. J., Muench, A. A., Rathborne, J., Alves, J. F., \& Lombardi, M. 2008, ApJ, 672, 410

Lada, C. J., \& Wilking, B. A. 1984, ApJ, 287, 610

Lee, W. C., \& Myers, P. C. 1999, ApJS, 123, 233

Li, P. S., Norman, M. L., Mac Low, M., \& Heitsch, F. 2004, ApJ, 605, 800

Luhman, K. L., Stauffer, J. R., Muench, A. A., Reike, G. H., Lada, E. A., Bouvier, J., \& Lada, C. J. 2003, ApJ, 593, 1093

Mac Low, M.-M., \& Klessen, R. S. 2004, Rev. Mod. Phys., 76, 125

Matzner, C. D., \& McKee, C. F. 2000, ApJ, 545, 364

McKee, C. F. 1989, ApJ, 345, 782

McKee, C. F., \& Zweibel, E. G. 1992, ApJ, 399, 551

Meyer, M. R., Adams, F. C., Hillenbrand, L. A., Carpenter, J. M., \& Larson, R. B. 2000, in Protostars and Planets IV, ed. V. Mannings, A. P. Boss, \& S. S. Russell (Tucson: Univ. Arizona Press), 121

Motte, F., André, P., \& Neri, R. 1998, A\&A, 336, 150

Muench, A. A., Lada, C. J., \& Rathborne, J. M. 2007, ApJ, 671, 1820

Muench, A. A., Lada, E. A., Lada, C. J., \& Alves, J. 2002, ApJ, 573, 366

Myers, P. C., Adams, F. C., Chen, H., \& Schaff, E. 1998, ApJ, 492, 703

Myers, P. C., \& Benson, P. J. 1983, ApJ, 266, 309

Nakano, T. 1998, ApJ, 494, 587

Nutter, D., \& Ward-Thompson, D. 2007, MNRAS, 374, 1413

Onishi, T., Kawamura, A., Tachihara, K., \& Fukui, Y. 2002, ApJ, 575, 950

Ossenkopf, V., \& Henning, Th. 1994, A\&A, 291, 943

Padgett, D. L., et al. 2008, ApJ, 672, 1013

Padoan, P., \& Nordlund, A.. 2002, ApJ, 576, 870

Rebull, L. M., et al. 2007, ApJS, 171, 447

Reid, I. N., Gizis, J. E., \& Hawley, S. L. 2002, AJ, 124, 2721

Rosolowsky, E. W., Pineda, J. E., Foster, J. B., Borkin, M. A., Kauffmann, J., Caselli, P., Myers, P. C., \& Goodman, A. A. 2008, ApJS, 175, 509

Salpeter, E. E. 1955, ApJ, 121, 161

Scalo, J. M. 1986, Fundam. Cosmic Phys., 11, 1 2005, in The Stellar Initial Mass Function Fifty Years Later, ed. E. Corbelli, F. Palla, \& H. Zinnecker (Dordrecht: Kluwer), 23

Schröder, K.-P., \& Pagel, B. E. J. 2003, MNRAS, 343, 1231

Shirley, Y. L., Evans, N. J., II, \& Rawlings, J. M. C. 2002, ApJ, 575, 337

Shirley, Y. L., Evans, N. J., II, Rawlings, J. M. C., \& Gregersen, E. M. 2000, ApJS, 131, 249

Shirley, Y. L., Huard, T. L., Pontoppidan, K. M., Wilner, D. J., Stutz, A. M., Bieging, J. H., \& Evans, N. J., II. 2007, ApJ, submitted

Shu, F. H. 1977, ApJ, 214, 488

Shu, F. H., Adams, F. C., \& Lizano, S. 1987, ARA\&A, 25, 23

Spezzi, L., et al. 2008, ApJ, 680, 1295

Spitzer, L., Jr. 1978, Physical Processes in the Interstellar Medium (New York: Wiley), 282

Tassis, K., \& Mouschovias, T. Ch. 2004, ApJ, 616, 283

Testi, L., \& Sargent, A. I. 1998, ApJ, 508, L91

Visser, A. E., Richer, J. S., \& Chandler, C. J. 2002, AJ, 124, 2756

Ward-Thompson, D., André, P., Crutcher, R., Johnstone, D., Onishi, T., \& Wilson, C. 2007, in Protostars and Planets V, ed. B. Reipurth, D. Jewitt, \& K. Keil (Tucson: Univ. Arizona Press), 33

Ward-Thompson, D., Scott, P. F., Hills, R. E., \& Andre, P. 1994, MNRAS, 268, 276

Young, C. H., Shirley, Y. L., Evans, N. J., II, \& Rawlings, J. M. C. 2003, ApJS, 145, 111

Young, K. E., et al. 2006, ApJ, 644, 326 (Paper II) 